\title{
AKUPUNKTUR IN DER PRÄVENTION, BEHANDLUNG VON VERLETZUNGEN UND VERBESSERUNG DER LEISTUNG BEI SPORTLERN: LITERATURBERICHT
}

\section{REVIEW ARTICLE}

JUNIOR, Julio Cesar Dias ${ }^{1}$

Junior, Julio Cesar Dias. Akupunktur in der Prävention, Behandlung von Verletzungen und Verbesserung der Leistung bei Sportlern: Literatur-Review. Revista Científica Multidisciplinar Núcleo do Conhecimento. 04-Jahr, Ed. 10, Band 10, $\begin{array}{llll}\text { S. } & \text { 59-98. } & \text { Oktober } 2019 . & \text { ISSN: }\end{array}$ Zugangslink: https://www.nucleodoconhecimento.com.br/gesundheit/akupunktur-inpravention

\section{ZUSAMMENFASSUNG}

Studien haben gezeigt, dass Akupunktur verbessert die körperliche Leistungsfähigkeit bei Profisportlern, aber das große Problem sind Sportverletzungen, die sie von

${ }^{1}$ Studium der Physiotherapie am Universitätszentrum araraquara - UNIARA - 2005 (Araraquara-SP); Lato sensu Postgraduiertenstudium in Orthopädischer und Traumatologischer Physiotherapie vom Cohen-Institut - Orthopädie, Rehabilitation und Sportmedizin - 2006 (Sao Paulo-SP)Ausbildung in Osteopathischer Manuellen Therapie durch das Cefisa Institut - 2008 (Araraquara-SP); Professional Improvement in Global Postural Reeducation and Sensory Motor Reprogramming - RPG/RSM by the Paulista Institute of Systemic Studies - IPES - 2010 (Ribeiréo Preto-SP); Lato sensu Graduiertenstudien in Systemischer Akupunktur vom Paulista Institute of Systemic Studies - IPES - 2013 (Ribeiréo Preto-SP); Stricto sensu Postgraduate Master es degree in Territorial Development and Environment from the University Center of Araraquara - UNIARA - 2016 (Araraquara - SP); Professionelle Verbesserung der klinischen und funktionellen Pilates durch das Paulista Institute of Systemic Studies - IPES - 2016 (Ribeiréo Preto-SP). 
Aktivitäten für längere Zeit verlassen können. Die Studie führte eine Literaturrecherche durch, die die Wirksamkeit der Technik bei der Prävention, Verbesserung der Leistung und bei der Behandlung von Läsionen anstrebte. Einige Autoren veranschaulichen und klären den Prozess der Schmerzneurophysiologie und die Art und Weise, wie Akupunktur in dieses Problem eingreifen kann, andere entwickeln und wenden Protokolle an, um eine Verbesserung der Leistung, Behandlung von Krankheiten und Prävention von Verletzung. Unter den Ergebnissen wurden 89 Artikel zu diesem Thema gefunden, wo: zweiundzwanzig berichteten über die Behandlung von Läsionen und 37 die Prävention und Verbesserung der sportlichen Leistung, die Anwendung einmal in der Woche, von 20 - 30 Minuten, zufriedenstellende Ergebnisse für die analysierten Daten. Basierend auf dieser Literatur und evidenzbasierten Praxis kann geschlossen werden, dass Akupunktur eine große Intervention ist, um diese Ziele im Bereich des Sports zu erreichen.

Stichworte: Akupunktur, Verletzungen, Schmerzen, Leistung.

\section{EINFÜHRUNG}

Seit mehreren Jahrtausenden Akupunktur wurde als eine Form der Prävention und Behandlung von verschiedenen Erkrankungen des Körpers verwendet. Diese Technik der chinesischen Medizin manipulierte Nadeln von Steinen und Fisch Pickel während der Steinzeit (3000 Jahre v. Chr.).1, 2 Der klassische Gelbe Kaiser: Huang Di Nei Jing, bildete eine der ältesten Aufzeichnungen der Traditionellen Chinesischen Medizin (MTC), Integration in die Gegenwart die philosophischen Grundlagen, die das Gleichgewicht von Universum und Natur konzeptualisieren, die in ihrer (chinesischen) Vision, Energie chi oder Qi genannt wird. Dieser Faktor hat zwei antagonistische Prinzipien, die abgeschlossen sind und dazu neigen, im Gleichgewicht zu sein, obwohl er unter dem Einfluss des Universums steht, genannt Yin und Yang. ${ }^{3}$

Akupunktur ist definiert als die Harmonisierung des Individuums mit der Umgebung, in der er ist, nicht nur, Nadeln einzufügen und das Problem der Krankheit zu beheben, aber das Ideal der Prävention von verschiedenen Pathologien zu erreichen.4, 5 Es ist ein Verfahren, das mit therapeutisches Ziel, ist nicht toxisch, ohne die Verwendung 
von chemischen Elementen, mit minimalen Nebenwirkungen, obwohl es in der Lage ist, endogene Substanzen wie Neurotransmitter freizusetzen. 4, 6, 7, 8, 9

Die Intervention soll die Akupunkte stimulieren, um die gewünschte therapeutische Wirkung zu erzielen und die Homöostase des Organismus zu erreichen. Viele Studien veranschaulichen die Auswirkungen der nicht-opioid-neuralen Theorie (hemmt Nervenimpulse, die auf das periphere und zentrale Nervensystem wirken); Humortheorie (stimuliert die Freisetzung von endogenen Opioiden, anderen Hormonen und Neurotransmittern).10, 11 Zweck in der Schmerzkontrolle, die Assoziation von Nadeln mit elektrischem Strom, genannt Elektroakupunktur, Potenziert und bietet Vorteile in der klinische Praxis, zusätzlich zu seiner Verwendung auch als: auricularakupunktur; Schädelpunktur; moxabusto; Saugnäpfe; Laserakupunktur. ${ }^{3,10}$

Im Sport nimmt der Einsatz von Technologie auf der Suche nach besseren Ergebnissen weiter zu und überschreitet die Grenzen des menschlichen Körpers. Mehrere natürliche oder integrative Therapien werden als Quelle der komplementären Therapie zur Verbesserung der sportlichen Bedingungen untersucht. Obwohl die Anzahl der Studien knapp ist, ist die Akzeptanz der Akupunktur immer noch sehr merkwürdig, da viele Menschen immer noch an den wissenschaftlichen Beweisen zweifeln. ${ }^{9}$ Jedoch hat seine Verwendung im sportlichen Umfeld zugenommen, indem sie bei der Behandlung von Schmerzen und sportlichen Verletzungen wirkt, beschleunigt den Prozess der Genesung des Individuums. Ein weiteres Thema ist der wirksame Eingriff in die Verbesserung der körperlichen Leistungsfähigkeit und die Verhinderung von Verletzungen durch Amateur- und Profisportler.12, 13, 14

\section{TOR}

Ziel dieser Arbeit war es, eine bibliographische Übersicht über den Einsatz von Akupunktur bei der Prävention, Verbesserung der sportlichen Leistung und bei der Behandlung von Verletzungen in verschiedenen Sportmodalitäten durchzuführen. 


\section{METHODIK}

Eine bibliographische Umfrage wurde in den Datenbanken Scielo, Google Acad'mico und Scoopus durchgeführt, um Informationen über: Akupunktur in der Prävention, Verbesserung der Leistung und bei der Behandlung von Sportverletzungen zu erhalten. Folgende Schlüsselwörter wurden verwendet: Akupunktur; Akupunktur und Schmerzen; Akupunktur in der Prävention; Sportverletzungen und Akupunktur; Akupunktur und Verbesserung der sportlichen Leistung; Behandlung von Verletzungen und Akupunktur.

Um den Ausschnitt dieser Studie abzugrenzen, wurde ein erstes Screening unter den zahlreichen gefundenen Artikeln durchgeführt, insgesamt 89 Manuskripte, die Auswahl von 22, die das Akupunktur-Thema bei der Behandlung von Verletzungen behandelt und 37 zur Prävention von Sportverletzungen und Verbesserung der sportlichen Leistung. Basierend auf dieser Analyse ist es erwähnenswert, dass von allen Studien, die in erster Linie gefunden wurden, das Universum der Forschung 59 Artikel umfasste, die in wissenschaftlichen Zeitschriften, Kongressnachlässen, Büchern, Websites veröffentlicht wurden, die im Titel, abstrakt oder Schlüsselwörter Terminologien, die während der Forschung verwendet werden.

\section{RESULTS UND DISCUSSION}

Bei dieser Suche ergaben sich mehrere Artikel (Schaubild 1; Tabelle 1), die isoliert durch die Schlüsselwörter ausgeführt wurde. 
Abbildung 1: Gesamtzahl der bei der Suche gefundenen und verwendeten Artikel.

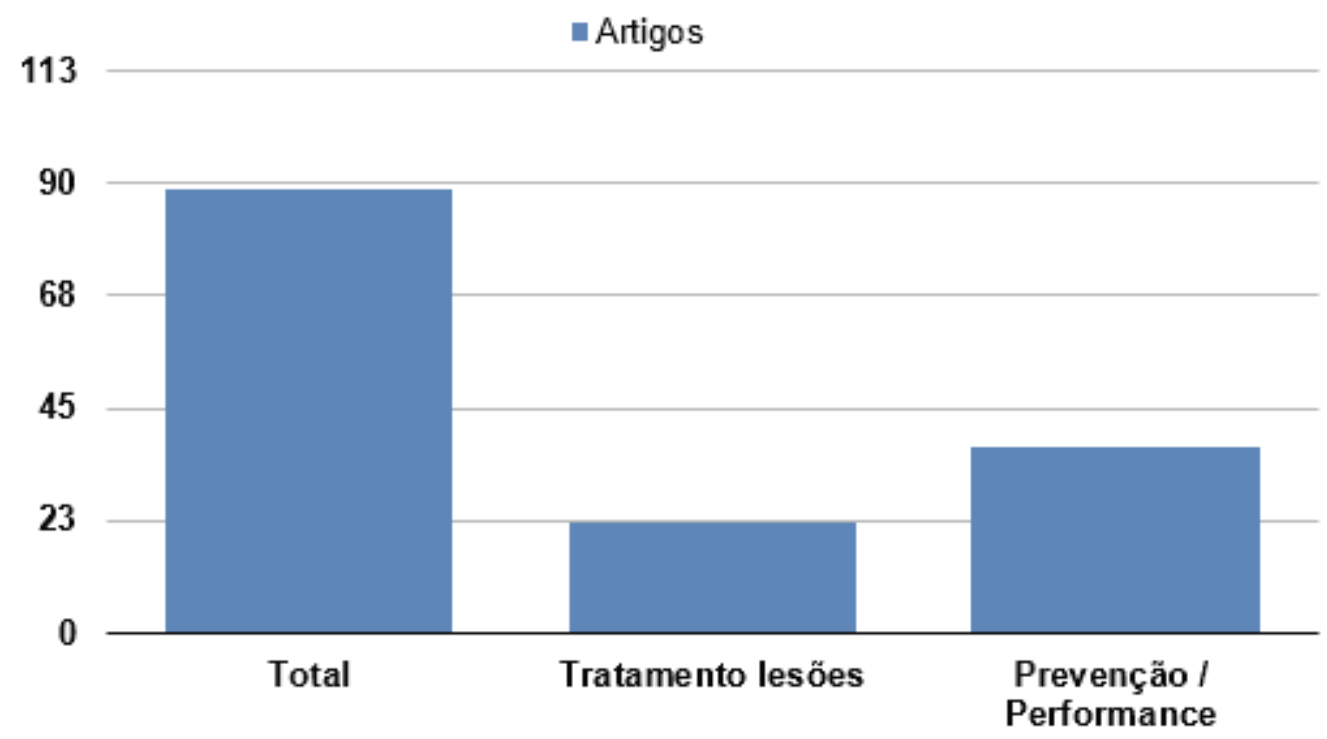

Tabelle 1: Artikel über Prävention, Verbesserung der sportlichen Leistung und Behandlung von Sportverletzungen.

\begin{tabular}{|c|c|c|}
\hline Autoren & Artikeltitel & Zeitschriften/Anais/Kongresse/Sites \\
\hline $\begin{array}{l}\text { Ehrlich D, Haber } \\
\text { P, } 1992\end{array}$ & $\begin{array}{l}\text { Influence of } \\
\text { acupuncture on pysical } \\
\text { performance capacity } \\
\text { and Haemodynamic } \\
\text { Parameters }\end{array}$ & J. Sports Med \\
\hline $\begin{array}{lr}\text { Santos } & \text { VC, } \\
\text { Kawano } & \text { MM, } \\
\text { Banja RA, 2008 }\end{array}$ & $\begin{array}{l}\text { Acupuntura na melhora } \\
\text { da performance em } \\
\text { atletas juvenis de } \\
\text { handebol }\end{array}$ & Rev Saúde e Pesq \\
\hline $\begin{array}{l}\text { Rubio K, Godoy } \\
\text { Moreira F, } 2008\end{array}$ & $\begin{array}{l}\text { A dor em corredores } \\
\text { com fascite plantar: o } \\
\text { uso da acupuntura }\end{array}$ & Rev Dor \\
\hline
\end{tabular}




\begin{tabular}{|c|c|c|}
\hline $\begin{array}{ll}\text { Pires } & \text { TF, } \\
\text { Pellegrinotti } & \text { IL, } \\
2010 & \end{array}$ & $\begin{array}{l}\text { Acupuntura na } \\
\text { Performance Atlética: } \\
\text { Estudo Exploratório }\end{array}$ & 8a Mostra Acadêmica da UNIMEP \\
\hline $\begin{array}{l}\text { Yang HY, Liu TY, } \\
\text { Gao M, } 2006\end{array}$ & $\begin{array}{l}\text { Electrical acupoint } \\
\text { stimulation increases } \\
\text { athletes rapid strength }\end{array}$ & Zhongguo Zhen Jiu \\
\hline $\begin{array}{l}\text { Luna } \\
\text { Fernandes } \\
\text { J, } 2005\end{array}$ & $\begin{array}{l}\text { Efeitos da Acupuntura } \\
\text { na performance de } \\
\text { Atletas velocista de alto } \\
\text { rendimento do Rio de } \\
\text { Janeiro }\end{array}$ & Fit e Perform J \\
\hline $\begin{array}{l}\text { Dias Junior JC, } \\
\text { Marino DM, } 2019\end{array}$ & $\begin{array}{l}\text { Acupuntura na } \\
\text { prevenção de lesões } \\
\text { musculares em atletas } \\
\text { de futebol profissional }\end{array}$ & Rev Fisiot S Fun \\
\hline Maciocia G, 2007 & $\begin{array}{l}\text { Os fundamentos da } \\
\text { medicina chinesa: um } \\
\text { texto abrangente para } \\
\text { acupunturistas } \\
\text { fitoterapeutas }\end{array}$ & Buch \\
\hline $\begin{array}{ll}\text { Akimoto } & \text { T, } \\
\text { Nakahori } & \text { C, } \\
\text { Aizawa } & \text { K, } \\
\text { Kimura } & \text { F, } \\
\text { Fukubayashi } & \text { T, } \\
\text { Kono I, 2003 } & \end{array}$ & $\begin{array}{l}\text { Acupuncture and } \\
\text { responses of imunoligic } \\
\text { and endocrine markers } \\
\text { during competition }\end{array}$ & Med Sci in Sports Exerc \\
\hline $\begin{array}{l}\text { Karvelas } \\
\text { Hoffman } \\
\text { Zeni Al, } 1996\end{array}$ & $\begin{array}{l}\text { Acute Effects of } \\
\text { Acupuncture on } \\
\text { Physiological and } \\
\text { Psychological }\end{array}$ & Arch Phys Med Rehabil \\
\hline
\end{tabular}




\begin{tabular}{|c|c|c|}
\hline & $\begin{array}{l}\text { Responses to Cycle } \\
\text { Ergometry }\end{array}$ & \\
\hline $\begin{array}{l}\text { Fry AC, Kraemer } \\
\text { KJ, } 1997\end{array}$ & $\begin{array}{l}\text { Resistance exercise ov } \\
\text { ertraing } \\
\text { and overreaching: neur } \\
\text { oendocrine responses }\end{array}$ & Sports Med \\
\hline Knardahl S, 1998 & $\begin{array}{l}\text { Sympathetic } \\
\text { nerve activity after } \\
\text { acupuncture in humans }\end{array}$ & $\begin{array}{l}\text { Department of } \quad \text { Clinical } \\
\text { Neurophysiology }\end{array}$ \\
\hline $\begin{array}{l}\text { Barlas PJ, } \\
\text { Robinson JA, Ba } \\
\text { xter GD, } 2000\end{array}$ & $\begin{array}{l}\text { Lack ofeffect of acupun } \\
\text { cture upon signs } \\
\text { and symptoms of delay } \\
\text { ed } \\
\text { onset muscle soreness }\end{array}$ & Clinical Physio \\
\hline $\begin{array}{l}\text { Rossetto SC, } \\
2009\end{array}$ & $\begin{array}{l}\text { Acupuntura nos } \\
\text { Esportes }\end{array}$ & Buch \\
\hline $\begin{array}{l}\text { França } \\
\text { Fernandes- } \\
\text { Senna V, Cortez } \\
\text { CM, } 2004\end{array}$ & 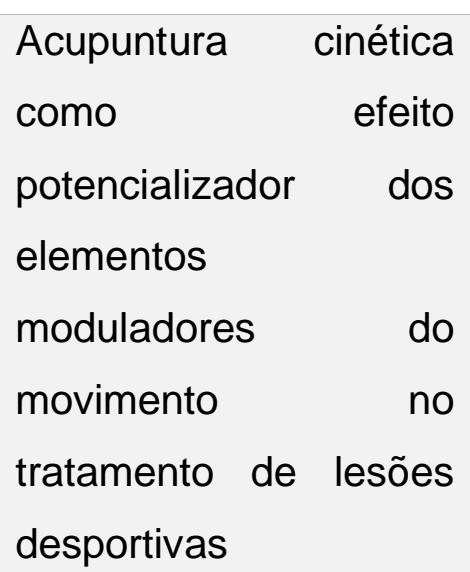 & Fisioter Bras \\
\hline $\begin{array}{l}\text { Wadsworth L T, } \\
2006\end{array}$ & $\begin{array}{l}\text { Acupuncture in sports } \\
\text { medicine }\end{array}$ & Curr Sports Med Rep \\
\hline Barela J A, 2000 & $\begin{array}{l}\text { Estratégias de Controle } \\
\text { em Movimentos } \\
\text { Complexos: Ciclo } \\
\text { Percepção - Ação no } \\
\text { Controle Postural }\end{array}$ & Rev Paul de Educ Fís \\
\hline
\end{tabular}




\begin{tabular}{|c|c|c|}
\hline $\begin{array}{l}\text { Papler PG et al., } \\
1999\end{array}$ & $\begin{array}{l}\text { Reabilitação do joelho. } \\
\text { In: Greve J.M. A. e } \\
\text { Amattuzzi, } \\
\text { Medicina de } \\
\text { reabilitação aplicada à } \\
\text { ortopedia } \\
\text { traumatologia }\end{array}$ & Buch \\
\hline $\begin{array}{l}\text { Gemeo LH. } \\
\text { Ignatti C, } 2004\end{array}$ & $\begin{array}{l}\text { Acupuntura como } \\
\text { Ferramenta Auxiliar do } \\
\text { Aumento da } \\
\text { Performance } \\
\text { Desportiva. In: Anais do } \\
\text { Simpósio Internacional } \\
\text { de Ciências Integradas } \\
\text { da UNAERP }\end{array}$ & Anais Simpósio \\
\hline Frasca L, 2011 & $\begin{array}{l}\text { Desempenho na Ponta } \\
\text { da Agulha }\end{array}$ & Rev Farmac \\
\hline Costa V, 2013 & $\begin{array}{l}\text { Acupuntura Previne } \\
\text { Lesões e ajuda a } \\
\text { melhorar } \\
\text { Desempenho nas } \\
\text { Pistas [periódico na } \\
\text { internet] }\end{array}$ & $\begin{array}{l}\text { http://blogs.oglobo.globo.com/pulso/ } \\
\text { post/acupuntura-previne-lesoes- } \\
\text { ajuda-melhorar-desempenho-nas- } \\
\text { pistas-500798.html. }\end{array}$ \\
\hline Luna M, 2016 & $\begin{array}{l}\text { Os Benefícios da } \\
\text { Acupuntura no Esporte }\end{array}$ & $\begin{array}{l}\text { http://www.ibramrp.com.br/noticia/49 } \\
\text { /os-beneficios-da-acupuntura-no- } \\
\text { esporte }\end{array}$ \\
\hline $\begin{array}{l}\text { Bopp-Limoge C, } \\
1998\end{array}$ & $\begin{array}{l}\text { L`acupuncture Permet- } \\
\text { elle D`ameliorer les } \\
\text { Performances } \\
\text { Sportives Stude } \\
\text { Personnelle a Propos }\end{array}$ & These Medicine \\
\hline
\end{tabular}




\begin{tabular}{|c|c|c|}
\hline & $\begin{array}{l}\text { de } 35 \text { Sportifs de haut } \\
\text { niveau. }\end{array}$ & \\
\hline Lin ZP et al., 2009 & $\begin{array}{l}\text { Effects of acupuncture } \\
\text { stimulation on recovery } \\
\text { ability for male elite } \\
\text { basketball athletes }\end{array}$ & Amer Jour of Chin Med \\
\hline $\begin{array}{l}\text { Lin, ZP et al., } \\
2009\end{array}$ & $\begin{array}{l}\text { Effect of auricular } \\
\text { acupuncture on oxygen } \\
\text { consumption of boxing } \\
\text { athletes }\end{array}$ & Chin Med Jour \\
\hline $\begin{array}{l}\text { Dhillon S et al., } \\
2008\end{array}$ & $\begin{array}{l}\text { The acute effect of } \\
\text { acupuncture on } 20-\mathrm{km} \\
\text { cycling performance. }\end{array}$ & Clin Jour of Spo Med \\
\hline $\begin{array}{l}\text { Hubscher M et } \\
\text { al., } 2010\end{array}$ & $\begin{array}{l}\text { Immediate effects of } \\
\text { acupuncture on } \\
\text { strength performance: a } \\
\text { ramdomized, controlled } \\
\text { crossover trial }\end{array}$ & Euro Jour of Appli Physi \\
\hline $\begin{array}{l}\text { Geng } L J \text { et al., } \\
1995\end{array}$ & $\begin{array}{l}\text { Investigation on the } \\
\text { effects of ear } \\
\text { acupressure on } \\
\text { exercise-induced lactic } \\
\text { acid levels and the } \\
\text { implications for athletic } \\
\text { training }\end{array}$ & Amer Jour of Acupu \\
\hline Franco RS, 2012 & $\begin{array}{l}\text { Avaliação do Efeito da } \\
\text { Acupuntura sobre o } \\
\text { Desempenho Físico } \\
\text { pelo Teste do Banco de } \\
\text { Harvard }\end{array}$ & Dissertação \\
\hline
\end{tabular}




\begin{tabular}{|c|c|c|}
\hline $\begin{array}{l}\text { Fonseca LP, } \\
\text { Lessa JFM, } 2011\end{array}$ & $\begin{array}{l}\text { Efeito da Aplicação da } \\
\text { Acupuntura na } \\
\text { Resistência Muscular } \\
\text { Localizada de Membros } \\
\text { Superiores em } \\
\text { Praticantes de } \\
\text { Exercício Resistido. }\end{array}$ & Mobografia \\
\hline $\begin{array}{lr}\text { Belmiro } & \text { H, } \\
\text { Vicentini } & \text { D, } \\
\text { Camilotti } & \text { CM, } \\
2013 & \end{array}$ & $\begin{array}{l}\text { Efeitos da Acupuntura } \\
\text { no Desempenho Motor } \\
\text { de Atletas. }\end{array}$ & Rev Fac Educ Fis \\
\hline $\begin{array}{l}\text { Rubio K; Godoy } \\
\text { Moreira F, } 2007\end{array}$ & $\begin{array}{l}\text { A representação da dor } \\
\text { em atletas olímpicos } \\
\text { brasileiros }\end{array}$ & Rev Dor \\
\hline Parisotto D, 2014 & $\begin{array}{l}\text { Efeito Imediato da } \\
\text { Aplicação da } \\
\text { Acupuntura na Dor } \\
\text { Muscular Tardia e na } \\
\text { Capacidade de } \\
\text { Contração Muscular }\end{array}$ & Dissertação \\
\hline Staud R, 2007 & $\begin{array}{l}\text { Mechanisms of } \\
\text { acupunture analgesia: } \\
\text { effective therapy for } \\
\text { musculoskeletal pain? }\end{array}$ & Curr rheumatol Rep \\
\hline $\begin{array}{l}\text { Bucinskaite } \mathrm{V} \text {, } \\
\text { Lundeberg T, } \\
\text { Stenfors C, } \\
\text { Ekblom A, Dahlin } \\
\text { L, Theodorsson } \\
\text { E, } 1994\end{array}$ & $\begin{array}{l}\text { Effects of electro- } \\
\text { acupuncture and } \\
\text { pysical exercice on } \\
\text { regional concentrations } \\
\text { of neuropeptides in rat } \\
\text { brain }\end{array}$ & Brain research \\
\hline
\end{tabular}




\begin{tabular}{|c|c|c|}
\hline Shang C, 2009 & $\begin{array}{l}\text { Prospective tests on } \\
\text { biological models of } \\
\text { acupuncture }\end{array}$ & $\begin{array}{l}\text { Evid Based Complement Alternat } \\
\text { Med. }\end{array}$ \\
\hline Bowsher D,1988 & $\begin{array}{l}\text { Mechanisms of } \\
\text { acupuncture. In: Filshie } \\
\text { J, White A. editors. } \\
\text { Medial Acupuncture: a } \\
\text { western scientific } \\
\text { approach. }\end{array}$ & Buch \\
\hline Ma SX, 2004 & $\begin{array}{l}\text { Neurobiology } \\
\text { acupuncture: } \\
\text { CAM }\end{array}$ & $\begin{array}{l}\text { Evid Based Complement Alternat } \\
\text { Med }\end{array}$ \\
\hline $\begin{array}{l}\text { Li J, Wang Q, } \\
\text { Liang H, Dong H, } \\
\text { Li Y, Ng EH, et al., } \\
2012\end{array}$ & $\begin{array}{l}\text { Biophysical } \\
\text { characteristics of } \\
\text { meridians and } \\
\text { acupoints: a systematic } \\
\text { review. }\end{array}$ & $\begin{array}{l}\text { Evid Based Complement Alternat } \\
\text { Med. }\end{array}$ \\
\hline $\begin{array}{l}\text { Lee, SH, Chung, } \\
\text { SH, Lee, JS, Kim, } \\
\text { SS, Shin, HD, } \\
\text { Lim, BV, et al., } \\
2002\end{array}$ & $\begin{array}{l}\text { Effects Acupunturaq on } \\
\text { the } \\
\text { hydroxytryptamine } \\
\text { synthesis and } \\
\text { tryptophan hydroxylase } \\
\text { expression in dorsal in } \\
\text { the dorsal raphe of } \\
\text { exercice rats. }\end{array}$ & Veurocienses Letters \\
\hline $\begin{array}{l}\text { Zyloney CE, } \\
\text { Jensen K, Polich } \\
\text { G, Loiotile RE, } \\
\text { Cheetham A, } \\
\text { LaViolette PS, et } \\
\text { al., } 2010\end{array}$ & $\begin{array}{l}\text { Imaging the functional } \\
\text { connectivity of the } \\
\text { Periaqueductal Gray } \\
\text { during genuine and } \\
\text { sham }\end{array}$ & Mol pain \\
\hline
\end{tabular}




\begin{tabular}{|c|c|c|}
\hline & $\begin{array}{l}\text { electroacupuncture } \\
\text { treatment. }\end{array}$ & \\
\hline $\begin{array}{l}\text { Minori AET, Mejia } \\
\text { DPM, } 2007\end{array}$ & $\begin{array}{l}\text { Atuação da Acupuntura } \\
\text { para o Tratamento de } \\
\text { LER/DORT no Ombro. }\end{array}$ & Monografia \\
\hline $\begin{array}{lr}\text { Brum } & \text { KN, } \\
\text { Alonso, } & \text { AC, } \\
\text { Brech GC, } 2009\end{array}$ & $\begin{array}{l}\text { Tratamento de } \\
\text { massagem em } \\
\text { acupuntura em } \\
\text { corredoresrecreacionai } \\
\text { s com síndrome do } \\
\text { piriforme }\end{array}$ & Arq Cienc Sau \\
\hline Hongwen S, 2003 & $\begin{array}{l}\text { Clinical Observation on } \\
\text { Acupuncture Treatment } \\
\text { of Piriformis Syndrome. }\end{array}$ & J Tradit Chin Med. \\
\hline $\begin{array}{lr}\text { Rocha } & \text { TBX, } \\
\text { Vilela Junior GB, } \\
\text { Martins } \\
\text { Manzatto } \\
\text { Grande AJ, } 2012\end{array}$ & $\begin{array}{l}\text { Análise Comparativa } \\
\text { Eletromiográfica do } \\
\text { Reto Femoral em } \\
\text { Isometria na Posição } \\
\text { Inferior do } \\
\text { Agachamento Wall } \\
\text { Slide, antes e após a } \\
\text { Aplicação } \\
\text { Acupuntura no Ponto } \\
\text { ST45 }\end{array}$ & Rev Bras Cien e Mov \\
\hline $\begin{array}{l}\text { Pinheiro RG, } \\
\text { Mejia DPM, } 2012\end{array}$ & $\begin{array}{l}\text { Efeito da Acupuntura na } \\
\text { Melhora do Paciente } \\
\text { com Quadro Álgico de } \\
\text { Lesão de Menisco } \\
\text { Medial. }\end{array}$ & Monografia \\
\hline $\begin{array}{l}\text { Nunes EA, Mejia } \\
\text { DPM, } 2012\end{array}$ & $\begin{array}{lr}\text { Tratamento } & \text { de } \\
\text { Acupuntura } & \text { para }\end{array}$ & Monografia \\
\hline
\end{tabular}




\begin{tabular}{|c|c|c|}
\hline & $\begin{array}{l}\text { Combater Dores nos } \\
\text { Ombros. }\end{array}$ & \\
\hline Tolentino F, 2016 & $\begin{array}{l}\text { Efeito de um } \\
\text { Tratamento com } \\
\text { Auriculoterapia na Dor, } \\
\text { Funcionalidade } \\
\text { Mobilidade de Adultos } \\
\text { com Dor Lombar } \\
\text { Crônica. }\end{array}$ & Dissertação \\
\hline
\end{tabular}

\section{ACUPUNCTURE IN DER PREVENTION VON INJURIES}

Die Prävention von Verletzungen im Sport ist ein großer Kampf, den Profis, die mit Athleten arbeiten, zu gewinnen. Im Fußball entspricht eine Muskelverletzung 20-40\% aller Sportverletzungen, überwiegend $80-90 \%$ in den unteren Gliedmaßen. ${ }^{15}$

Muskelverletzung ist der große Bösewicht aller Athleten, aus verschiedenen Sportarten, und kann sie von ihren Aktivitäten für eine lange Zeit verlassen. Daher ist es interessant, einen Vorschlag für eine prophylaktische Intervention vorzulegen, die die Idee einer Technik zur Vorbeugung oder Verringerung der Risiken dieser Art von Verletzungen bei Sportlern mitbringt. In diesem Fall erweist sich Akupunktur als eine vorteilhafte Technik.

In seiner Studie, Dias Junior 16, wurde von 54 männlichen Athleten besucht, die zu einer professionellen Fußballmannschaft gehören. Sie wurden in 6 Gruppen verteilt: Gruppe 1 - Akupunktur, um das System neu auszubalancieren, durch Auswertung gefunden; Gruppe 2 - die Protokoll mit spezifischen Punkten erhielt: Milz-Pankreas 3, Milz-Pankreas 6 (Abbildung 1A) und Magen 36 (Abbildung 1B); Gruppe 3 - Magen 36 (Abbildung 1B); Gruppe 4 - Milz Bauchspeicheldrüse 6 (Abbildung 1A); Gruppe 5 Ohrakupunktur; Kontrollgruppe - erhielt keine Intervention. Nach acht Sitzungen, eine pro Woche, zeigten die Ergebnisse: Die Gruppen 1 und 2 zeigten keine Art von Verletzungen; Gruppe 4: zwei Läsionen; Gruppen 3 und 5: präsentiert 1 Läsion; Kontrolle: 6 Fälle von Muskelproblemen dargestellt. 
Abbildung 1 A: Milz-Bauchspeicheldrüse 3 - auf der medialen Seite des Fußes, posttero-unterlegen des Metatarsal-Phaline-Gelenks, in der Linie der Kreuzung von dunkler und heller Haut; Bauchspeicheldrüse Milz 6: 3 Tsun über dem medialen Maleolus, am posteromedialen Rand der Tibia; B: Magen 36 - 3 Tsun unter der Patella zwischen dem vorderen Tibiamuskel und dem langen Extensormuskel der Finger; Magen 37 - 3 Tsun unter Zusanli (E36), auf der seitlichen vorderen Seite des Tibiamuskels; Magen 38 - 8 Tsun unter dem Knie, 2 Tsun unter dem Punkt Shangjuxu (E37), auf der Seite des vorderen Tibiamuskels; Magen 41 - an der dorsalen Mitte des Knöchels über dem Kreuzband, zwischen den Sehnen des langen HalluxExtensormuskels und dem langen Extensor der Finger.

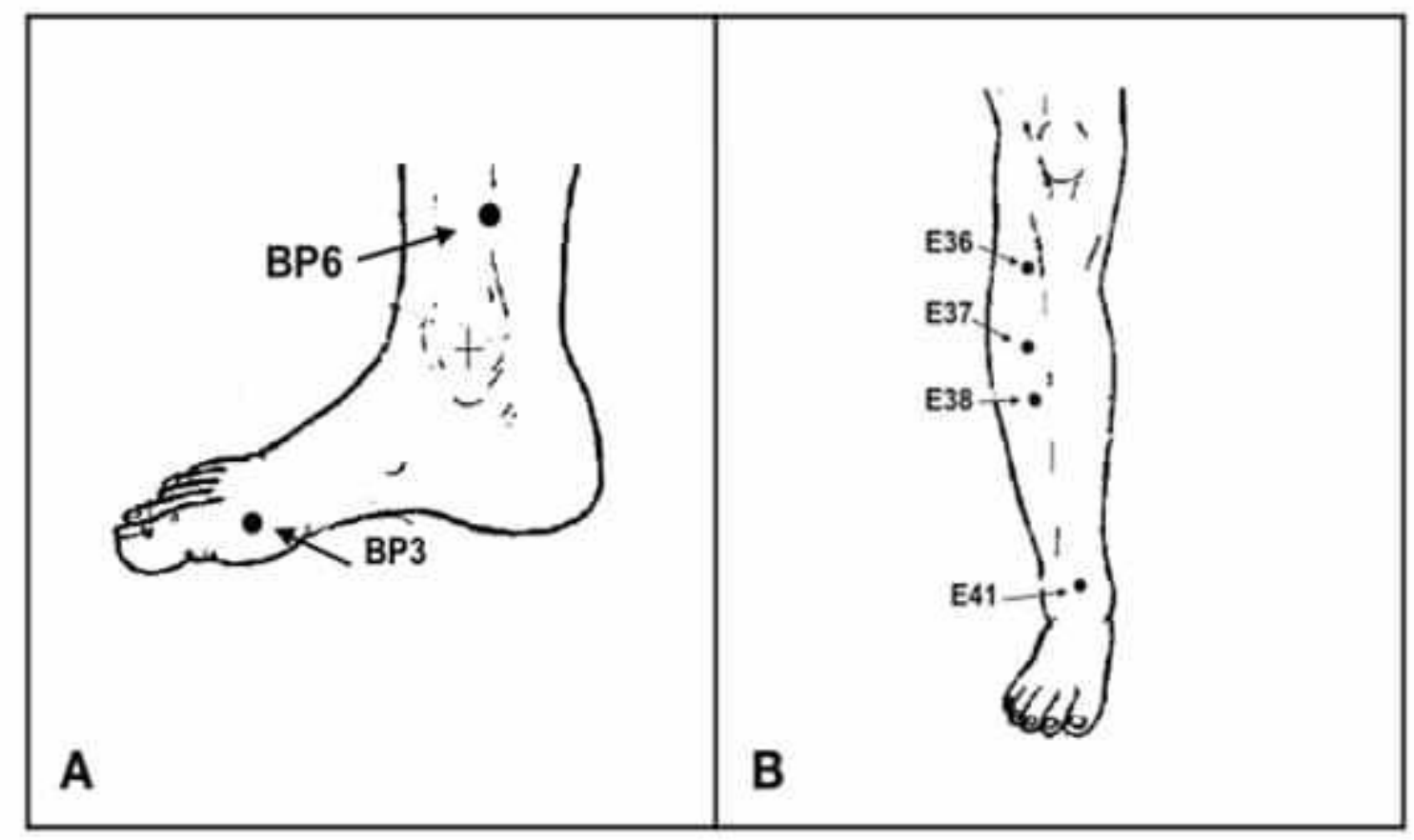

Quelle: Wen ${ }^{17}$

Die Bauchspeicheldrüse Milz hat die Funktion, Energie aus der Nahrung zu extrahieren, an den Körper zu verteilen und Blut in den Blutgefäßen zu kontrollieren. Die starke Präsentation Qi wird zu allen Muskeln im Körper transportiert werden, speziell zu den Gliedmaßen, aber wenn das Qi in Mangel ist, wird die Energie nicht auf Muskelgewebe übertragen und der Athlet wird Müdigkeit und geschwächte Muskeln präsentieren ${ }^{18}$ 
Nach Ross 19, die Milz Punkte Bauchspeicheldrüse 3 und 6 sind mit Magen 36 durch Tonnnierung Blut Qi verbunden. Das Erdelement ist verantwortlich für die Energiebilanz und die Verfügbarkeit von Qi und Blut als Energiequellen für geistige, emotionale und körperliche Aktivität. THE MTC, berichtet, dass durch die Tonung dieses Elements, es hält die Muskeln von Qi und Xue genährt (Blut in DER MTC), mit dem Ziel, die sportliche Leistung zu verbessern. Dies behält die Pflicht, das Organ, das Xue (Leber) produziert und hält die energetische Flüssigkeit (Spleen Pankreas und Niere), da die Funktionalität des Blutes ist, den Körper zu nähren, zusätzlich zur Vollendung der Nährstoffwirkung von Qi.

Muskeltraining, Entspannung und kontraktile Kraft ist mit Leber xue Ernährung verbunden. Wenn die Ernährung mangelhaft ist, werden Krämpfe und Parästhesien der Gliedmaßen Verletzungen verursachen. Auf der anderen Seite überwacht Milz das Xue in den Gefäßen und entfernt Qi von der Übertragung von Lebensmitteln an den Körper: Starke Qi, wird gute Energie an die Muskeln übertragen; ein geschwächtes Qi führt zu einem müden und schwachen Muskel. ${ }^{19,} 20$

Basierend auf diesen Erkenntnissen wird diese Technik in Sportarten praktiziert, die schnelle Ergebnisse präsentieren, verbesserungder Qualität der Muskelkraft, herzkreislauf-Bedingungen, Flexibilität und geistiges und körperliches Wohlbefinden der Sportler. 20, 21, 22 Yang und 22 Mitarbeiter, erreichteine deutliche Erhöhung der Muskelkraft, die Verbesserung der Geschwindigkeit der Athleten. Luna und die Mitarbeiter 24 wiederum erklärten, dass die Stimulation von Akupunkturpunkten die sportliche Leistungsfähigkeit verbessert, Muskelplastizität, die Verletzungen verhindert.

Eine in Japan durchgeführte Studie zeigt die Auswirkungen der Akupunktur auf das körperliche Wohlbefinden von Fußballerinnen während des Wettbewerbs auf. Sie wurden in zwei Gruppen eingeteilt, in denen eine der Behandlung und die andere Kontrolle unterzogen wurde. Cortisolspiegel (endokrine Systembewertungen) und die Beurteilung des körperlichen Wohlbefindens wurden anhand des POMS-Fragebogens (Bewertet des physischen und geistigen Status) bewertet: Es wurde eine Verbesserung des Immunsystems beobachtet ( verminderte SlgA-Sekretion), 
verbesserte psychische Belastung (vermindertes Cortisol) und verbessert das körperliche Wohlbefinden, Flexibilität und Muskelspannung. ${ }^{25}$

Ehrlich und 25 Mitarbeiter erreichte ${ }^{n}$ jedoch eine Verbesserung der sportlichen Leistung, die Steigerung des körperlichen Wohlbefindens, die Verbesserung der Wettkampfreaktion, während des Trainings und der Spiele, die Unterstützung bei der Erholung der Muskelkapazität.

Andere Läsionen, die mit Akupunktur verhindert werden können, sind Knöchelverstauchungen, die die Bänder beeinflussen, viele Schmerzen und Einschränkung der Gelenkfunktion auslösen. France $2^{6}$, verwendete die Ashi-Punkte (schmerzhafte Punkte zur Palpation), die mit Blase 60 assoziiert sind (Abbildung 2A), um Schmerzen zu beseitigen; die Gallenblase 39, die die Knochen, Sehnen verstärkt und die Algie in den Extremitäten bekämpft; und Gallenblase 40 stimuliert Qi und Xue; Magen 41 (Abbildung 2B), der seitliche Veränderungen des Knöchels und des Magens 36 behandelt (Abbildung 1B), die Obstruktion beseitigen und die Durchblutung von Qi und lokalem Xue stimulieren.

Abbildung 2 A: Blase 60 - zwischen der Achillessehne und dem Rand des seitlichen Soleolus des Knöchels, auf der Höhe des höchsten Punkts des Maleolus; B: Gallenblase 39 - 3 Ssun über dem höchsten Punkt des äußeren Maleolus, bei Depression zwischen Fibel und langen und kurzen fibulären Sehnen und Gallenblase 40 - auf der antero-unterlegenen Seite des äußeren Maleolus, bei seitlicher Depression der Sehne des langen digitalen Extensormuskels ; Magen 41 - an der dorsalen Mitte des Knöchels über dem Kreuzband, zwischen den Sehnen des langen Halux-Extensormuskels und dem langen Extensor der Finger.

Quelle: Wen 17

Einige Athleten können mit Verstauchung überrascht sein, die als eine Halssehnenverletzung definiert ist, die Muskelschmerzen und Steifheit verursacht. Es gibt ein Protokoll, um dieses Problem zu beheben und den Athleten zu seinen Aufgaben zurück. Wadsworth ${ }^{27}$, verwendet zusätzlich zu den Ashi-Punkten, die die 
Zirkulation von Qi und Xue stimulieren, die Gallenblase 39 (Abbildung 2B), Gallenblase 20 wirkt in der Zirkulation von Qi und Xue, entspannende Sehnen und erweichen Schmerzen; Gouverneur ousse 14 die Ausweisung des pathogenen Faktors und die Regulierung des Meridians Qi; Blase 10; Dünndarm 14; Gallenblase 21 (Abbildung 3A) stimulieren die Durchblutung von Qi und Xue. Wenn der Gelenkblock Die Beugung und Ausdehnung begrenzt, verwenden Sie Blasenpunkt 60 (Abbildung 2A). Dünndarmpunkte 3, Lunge 7 fördern die Durchblutung von Qi und Xue des Halses, wenn es Rotationsbeschränkung hat, verwenden Sie den Dünndarmpunkt 7, der den Taiyang Meridian der Hand entwässert (Abbildung 3B).

Abbildung 3 A: Gallenblase 20 - unterhalb der okzipitalen Kante in Depression zwischen Trapez- und Sternum-Clidomastoiden-Muskeln, am Rande der Haare und Gallenblase 21 - am äquidistanten Punkt zwischen dem Dazhui (VC14) und dem Schulteracromion, 1 Tsun über dem tianian Punkt; Gouverneurvase 14 - in der Mitte zwischen den dornigen Prozessen; siebter Halswirbel und erster Rückenwirbel; Blase 10 - auf der Ebene zwischen den Stacheln des zweiten und dritten Wirbels, 1,3 seitliche Sonnentau der dorsalen Mittellinie, an der Randseite des Trapezmuskels und dünndarm 14 - 3 seitliche Tsun der Achse des Wirbels; auf der horizontalen Ebene der unteren Kante des dornigen Prozesses des ersten dorsalen Wirbels; B: Lunge 7 - auf der medialen Seite des Unterarms, 1,5 Tsun über der Handgelenklinie zwischen den Sehnen des langen Daumenadduktorenmuskels und dem langen Extensormuskel carporadial; Dünndarm 3 - ulnarseite der Hand, hinter dem metakarpal-phalangischen Gelenk des fünften Fingers, zwischen der hellen und dunklen Haut; Dünndarm 7 - 5 tsun über dem Handgelenk, auf der ulnaren Seite des Karpal-Ulnar-Extensormuskels.

Quelle: Wen 17

Überlastung des Ellenbogengelenks, wirkt, vor allem Tennisspieler, und kann zu einer Blockade von Qi und Xue führen, die eine chronische Entzündung namens Epicondilite auslöst. Ashi-Punkte werden in der Region verwendet, die Qi-Stimulation fördert. ${ }^{28}$

Zur Behandlung von Handgelenkläsionen, wie: Sehnenentzündung oder Tenosynovitis des Daumens, Extensoren und Flexoren der Finger, Franc ${ }^{e}$ 26, 
verwendet die Ashi-Punkt mit dem Dickdarm 5 verbunden, um die Wärme des Meridians zu zerstreuen; Dünndarm 5, wodurch Wärme entfällt und das Handgelenk entriegelt wird (Abbildung 4A); Dreifachheizung 4, die neben der Beseitigung von Hitze beendet Entzündungen und der Dickdarm 11 (Abbildung 4B) Entriegelung Qi und Xue Beruhigung der Schmerzen.

Abbildung 4 A: Dünndarm 5 - auf der ulnaren Seite des Handgelenks, in der Depression zwischen der Pisiform und dem Ulnar-Styloid-Prozess; Dickdarm 5 - auf der Rückseite-radiale Seite des Handgelenks, ein wenig distal des Radioknochens, wo es Depressionen zwischen den Sehnen des Extensormuskels kurz und lang des Daumens, beim Dehnen und Öffnen des Daumens. B: Dreifachheizung 4 - auf der Rückenseite des Handgelenks, in der Depression in der Mitte der Rückenfalte des Handgelenks, zwischen den Sehnen der gemeinsamen digitalen Extensormuskulatur und dem digitalen Extensor des fünften Fingers; Dickdarm 11 - auf der radialen Seite des Ellenbogens, im brachioradialen Muskel; beim Biegen des Ellenbogens, in radialer Vertiefung am Ende der Ellenbogenlinie.

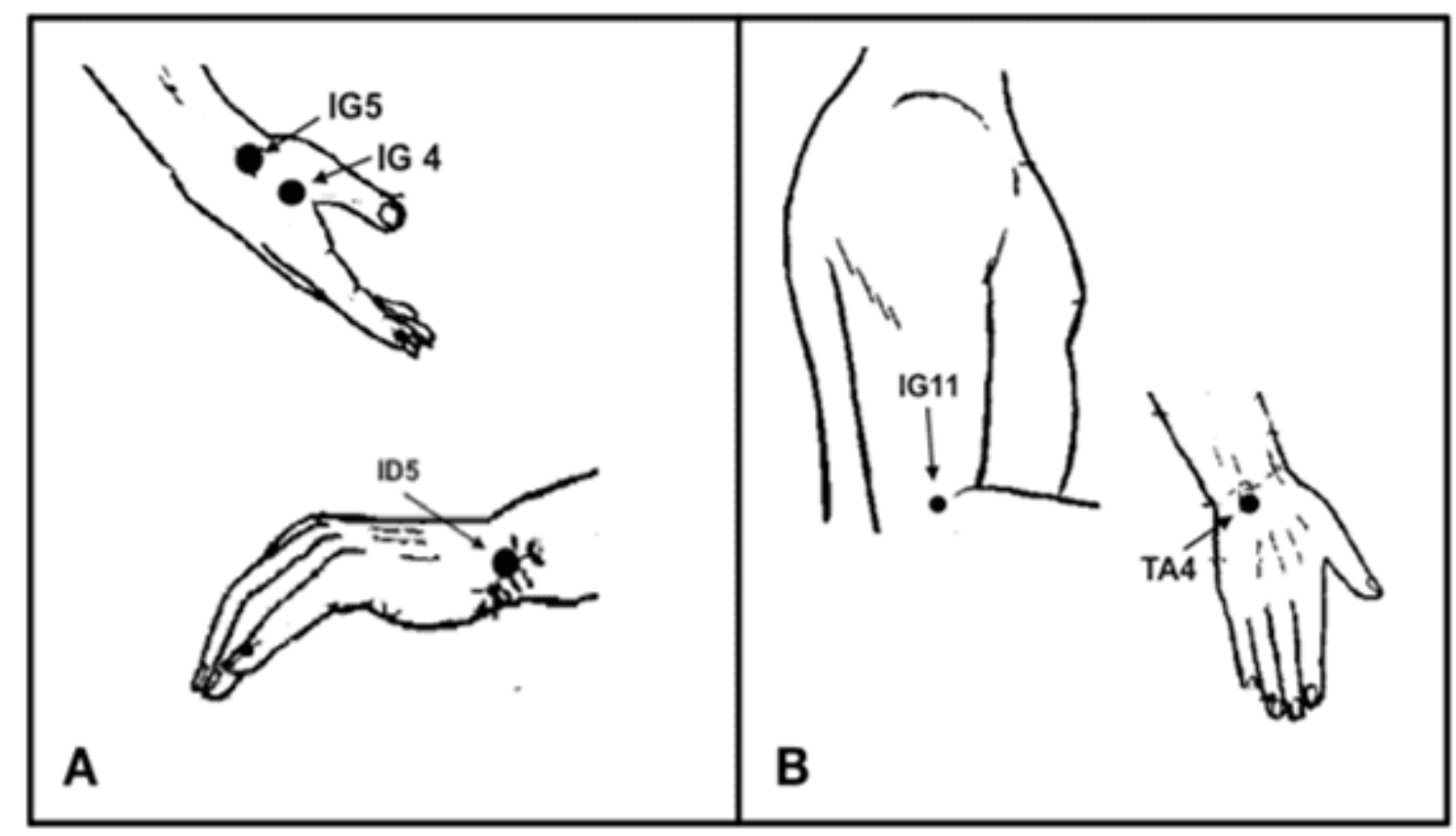

Quelle: Wen ${ }^{17}$ 
Für Schmerzen im unteren Rückenbereich, Barela 17, assoziiert eshi Punkte mit Blase 23 und Blase 25, um lokale Qi und Xue zu stimulieren, Blase 40 (Abbildung 5), die Schmerzen und Hitze beseitigt.

Abbildung 5: Blase 23 - 1,5 Tsun, seitlich am unteren Rand des Wirbelsäulenprozesses des Wirbels (L2); Blase 25 - 1,5 Tsun, seitlich am unteren Rand des Wirbelsäulenprozesses des Wirbels (L4); Blase 40 - 3 Tsun, seitlich der Achse (Du-Mai), an der unteren Randebene in der zweiten Wirbelsäule des Wirbels (T2).

Quelle: Wen 17

Nun ist eine weitere Verletzung, die Fußballsportler beunruhigt, Bänder, Meniscals und Sehnen des Knies. Dies wird mit Ashi-Punkten behandelt, verbunden mit Gallenblase 33 (Abbildung 6), die Sehnen entspannt und Schmerzen behandelt; Blase 40 (Abbildung 5) beseitigt Gelenkschmerzen, Ödeme und Hitze und stimuliert Qi und Xue; Magen 36 (Abbildung 1B) reguliert den Fluss von Qi und Xue des Meridians und beseitigt Obstruktion. ${ }^{29}$

Abbildung 6: Gallenblase 33 - am seitlichen Rand des Knies, 3 Sonnen über dem Yanglingquan-Punkt (VB34), in der oberen Depression des lateralen Epithenodilofs des Oberschenkelknochens.

Quelle: Wen 17

Gemeo ${ }^{30}$, erklärte, dass Akupunktur kann eine Verbesserung der körperlichen Leistungsfähigkeit der Athleten fördern, verhindern und behandeln Verletzungen, die vor, während oder nach Sportwettkämpfen auftreten können, zusätzlich zur Unterstützung und Beeinflussung von emotionalen Faktoren wie Angst, Reizbarkeit, Schlaflosigkeit und Depression, die die sportliche Leistungsfähigkeit dieser Personen direkt behindern können. Er entwickelte ein spezifisches Protokoll mit MasterAkupunkturpunkten, das nach der Modalität auswählt (Abbildung 1): 
Tabelle 2: Gemeo ${ }^{30}$

\begin{tabular}{|c|c|}
\hline $\begin{array}{l}\text { Meister der moralischen } \\
\text { Energie }\end{array}$ & Gouverneur Vase 19 (Abbildung 7) \\
\hline $\begin{array}{l}\text { Meister der allgemeinen } \\
\text { Energie }\end{array}$ & $\begin{array}{l}\text { beeinflusst die allgemeine Energie, die auf die } \\
\text { Ahnen- und postnatale Energie einwirkt - Die } \\
\text { Vase des Gouverneurs 4, 6, 10,13 (Abbildung 7) }\end{array}$ \\
\hline $\begin{array}{l}\text { Meister der oberen } \\
\text { Gliedmaßen }\end{array}$ & $\begin{array}{l}\text { wirkt auf Unterarmbewegungen, Handgelenke, } \\
\text { Hände und Schultern: Dreifachheizung } 15 \\
\text { (Abbildung 7) }\end{array}$ \\
\hline Meister der Lunge & stört den vorderen Thorax: Lunge 1 (Abbildung 7) \\
\hline Zurück-Master & $\begin{array}{l}\text { Zwerchfell, Atmung und nn. Phrenonisch: } \\
\text { Blase17 (Abbildung 7) }\end{array}$ \\
\hline Bauchmeister & $\begin{array}{l}\text { Einfluss auf die Bauchtaille: Bauch } 27 \text { (Abbildung } \\
\text { 7) }\end{array}$ \\
\hline Meister der Nieren & $\begin{array}{l}\text { Lendenwirbelsäule: Blase } 47 \text { und Blase } 52 \\
\text { (Abbildung 8) }\end{array}$ \\
\hline Hüftmeister & $\begin{array}{l}\text { wirkt die } \mathrm{mm} \text {. Glutes und Coxofemoralgelenk } \\
\text { (Abbildung 8) }\end{array}$ \\
\hline Oberschenkelmeister & $\begin{array}{l}\text { in der Muskulatur der Oberschenkel: Gallenblase } \\
30 \text { (Abbildung 8) }\end{array}$ \\
\hline Untere Gliedmaßenmeister & $\begin{array}{l}\text { beeinflusst die Wirkung von Füßen und Muskeln } \\
\text { im Allgemeinen: Blase } 58 \text { (Abbildung } 8 \text { ) und } \\
\text { Magen } 36 \text { (Abbildung 1B) }\end{array}$ \\
\hline $\begin{array}{l}\text { Punkte zur Verbesserung von } \\
\text { Gleichgewicht und Reflexion }\end{array}$ & $\begin{array}{l}\text { vaso-Konzeption 4, Vaso-Konzeption } 6 \\
\text { (Abbildung 9), Dickdarm } 11 \text { (Abbildung 4B), Blase } \\
46 \text { (Abbildung 8), Regler Gefäß } 4 \text { (Abbildung 7). }\end{array}$ \\
\hline
\end{tabular}

Abbildung 7: Vasen des Gouverneurs 4 - in der Mittellinie der Wirbelsäule; im Raum zwischen den Stacheln des zweiten und dritten Lendenwirbels; Gouverneur Vase 6 in der Mittellinie der Säule; zwischen dem elften und zwölften dornigen Prozess der Dorsalwirbel; Gouverneur Vase 10 - in der Mittellinie der Säule; zwischen dem 
sechsten und siebten dornigen Prozess der Dorsalwirbel; Gouverneurvase 13 - in der Mittellinie der Säule; zwischen dem ersten und zweiten dornigen Prozess der Dorsalwirbel; Gouverneursvase 19 - 1,5 Tsun hinter Baihui (VG 20); Magen 27 - 2 Tsun unter dem Nabel und 2 Tsun neben der medialen Linie am seitlichen Rand des Rectus-Bauchmuskels; Blase 17 - 1,5 Tsun der Achse, auf der unteren Randebene des Spinalprozesses des Wirbels (T7); Lunge 1 - auf der anterolateralen Seite der Brust, unterhalb des Yunmen-Punktes (P2) (Grube zwischen Schlüsselbein und Schulter), im Raum zwischen der ersten und zweiten Rippe, 6 Ssun auf der Mittellinie des Körpers; Dreifachheizung 15 - in der supraskaporären Grube, zwischen Quyuan (ID13) und Jianjing (VB21).

Quelle: Wen 17 
Abbildung 8: Blase 46 - 3 Tsun, seitlich der Achse, am unteren Rand der Wirbelsäule (T9); Blase 47 - 3 Tsun, seitlich der Achse, am unteren Rand der Wirbelsäule (T10); Blase 52 - 3 Tsun, seitlich der Achse, auf der Ebene der zweiten Wirbelsäule des Kreuzbeins, am seitlichen Rand des iliossakralen Gelenks; Blase 58 - I tsun unter der Seite des Chengshan-Punktes (B57); 7 Tsun über der Ferse, auf der Seitenseite der Gastrocnemius Muskelsehne; Gallenblase 30 - im Gesäß, in der Linie zwischen der heiligen Pause und der prominenten des größeren Trochanter; ein Drittel des seitlichen Abstands am unteren Rand des piriformen Muskels; extra Hüftpunkt - in der Mitte der Linie, die vom oberen Ende der interglutealgroove bis zum Teil des äußeren Kamms des Ischio reicht.

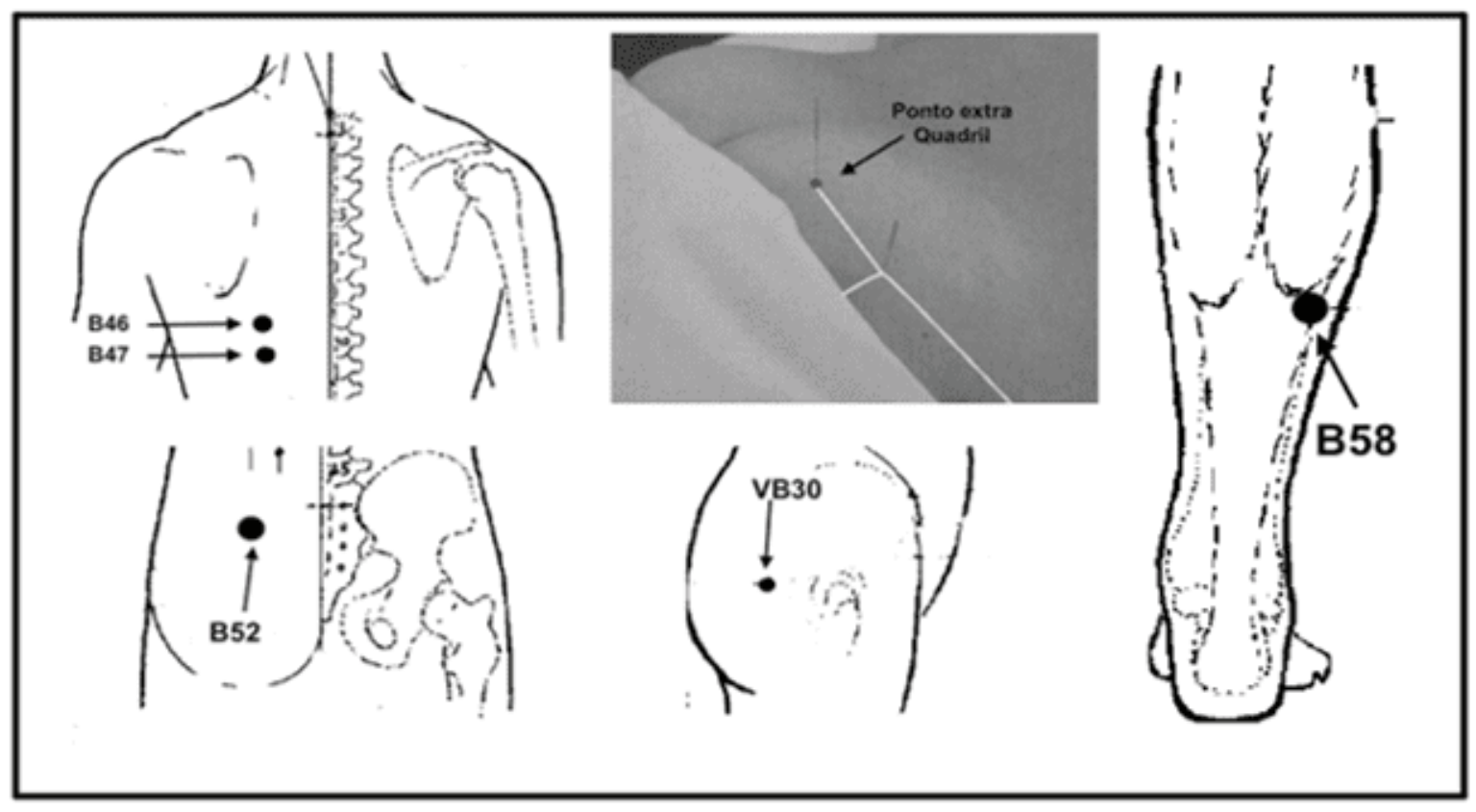

Quelle: Wen ${ }^{17}$; Rosseto ${ }^{31}$

Abbildung 9: Vasenkonzeption 4 - 3 Tsun unter dem Nabel, in der Mittellinie des Bauches; Vase Konzeption 6 - 1,5 Tsun unter dem Nabel, in der Mittellinie des Bauches.

Quelle: Wen 17 


\section{ACUPUNCTURE IN DER VERBESSERUNG DER ATHLETIC PERFORMANCE}

Bei der Akupunktur bei der Prävention von Verletzungen bei Sportlern dürfen wir nicht vergessen, dass wir bei der Erreichung dieses präventiven Ziels folglich die Qualität und körperliche Unversehrtheit verbessern und in die Leistungssteigerung eingreifen können.

In asiatischen Ländern entwickelt die Verwendung von Akupunktur ein körperliches Wohlbefinden für Sportler, reguliert durch nervöses, endokrines Immunsystem und als Ergebnis, eine Verbesserung der körperlichen Verfassung in mehreren Modalitäten. ${ }^{32}$, ${ }^{31}, 34$ Darüber hinaus gibt es Punkte vor, während und nach dem Training und Spiele, die bei der Erhaltung der Muskeln helfen, wie bei der Verringerung der Milchsäure, Verringerung von Schmerzen, Müdigkeit, Beschleunigung der Erholung und Verbesserung der Leistung. ${ }^{35}$

Akupunktur stimuliert die Freisetzung von biochemischen Substanzen, die Muskelermüdung von intensiven Übungen lindert, neben der Förderung erhöhter antioxidativer Aktivität, Verringerung der oxidativen Stressraten. Während einer Studie erhielten die Athleten eine Behandlung mit Elektroakupunktur für 30 Minuten, über einen Zeitraum von 30 Tagen und das Ergebnis war die signifikante Zunahme der Superoxid-Dismutase (SOD) und verminderte Blutmalondial (MDA). SuperoxidDismutase ist ein Enzym mit antioxidativer Wirkung, das Zellen vor toxischen Substanzen schützt, indem es eine starke entzündungshemmende Reaktion im Körper ausübt. Der MDA ist mit entzündlichen und degenerativen Erkrankungen verbunden, so dass die Forscher zu dem Schluss kommen können, dass Elektroakupunktur die MDA-Raten senkt und die SOD erhöht, was zur Ermüdungsentlastung und zur Verbesserung der körperlichen Leistungsfähigkeit beiträgt. ${ }^{36}$

Obwohl es eine jahrtausendewährende Technik ist, verbessert seine Verwendung die körperliche und geistige Leistungsfähigkeit des Athleten ist neu. Luna veröffentlichte eine Studie, die zeigte, dass Akupunktur statistisch die maximale Stärke und Potenz 
von Sprinter-Athleten (100, 200 und 400 Meter flach ohne Barrieren) verbessert und seitdem die Forschung in dieser Hinsicht vertieft wurde. ${ }^{37}$

In einer Forschung, $\mathrm{S}^{\text {antos }} 20$ verwendet männliche Handbol Athleten zwischen 15 und 17 Jahre alt, wo er zwei 100-Meter-Schüsse in jedem Athleten (Vor-Akupunktur) durchgeführt. Nach 45 Minuten Anwendung des für die Behandlung gewählten Protokolls hat er auf die gleiche Weise neu bewertet. Die ausgewählten Punkte waren: Blase 58 (Abbildung 8) Meister der Beine und des Fußes; Magen 36 (Abbildung 1B) strafft die gestreifte Muskulatur und wird verwendet, um die Behandlung zu potenzieren; zusätzlicher Hauptpunkt der Hüften (Abbildung 8) strafft die Gesäße und erhöht die Flexibilität des Coxofemoralgelenks; Gallenblase 30 (Abbildung 8) Meister der Oberschenkel und fördert die Flexibilität für die Bewegung der unteren Gliedmaßen; Lungen-1-Träger der Lunge (Abbildung 7) fördert effiziente Atemarbeit; Blase 17 (Abbildung 7) Meister des Rückens, dorsale Zwerchfell shu und Xues Einflusspunkt, der den Atemrhythmus reguliert; Dreifachwärmer 15 (Abbildung 7) Meister der Arme, die die gemeinsame Muskelgruppe von Schultern, Nacken, Halswirbelsäule, ersten sechs Brustwirbeln, Armen, Unterarmen, Handgelenken und Hand befehligt.

Die Ergebnisse ergaben eine signifikante Verbesserung, mit einer Abnahme der Zeit in der zweiten Batterie der 100-Meter-Tests. Die durchschnittliche Verbesserung in der Zeit war 5,72\% und der Athlet mit der besten erreichten Leistung erreicht einen Prozentsatz von $10,84 \%$.

Dieser Durchschnitt von 5,72\% in der Verbesserung der Leistung erreicht scheint klein, aber im Vergleich zu den Ergebnissen der wichtigen Wettbewerbe, können wir die Gültigkeit der Praxis zu sehen: bei den Panamerikanischen Spielen in Rio de Janeiro 2007, im Finale der 100 Meter flachen männlichen Der Unterschied zwischen dem 1. Platz und dem 8. Platz betrug 0,23 Sekunden, was einer Umzeit von 2,26\% entspricht, d.h. wenn dieser Athlet eine Verbesserung von 5,72\% in seiner Leistung erreichen würde, würde er wahrscheinlich andere Ergebnisse erzielen. 
Es gibt wenig wissenschaftliche Aufmerksamkeit, wenn wir behandeln, von Akupunktur-Effekte auf Verbesserungen in der physiologischen Reaktion durch Bewegung induziert und nur wenige gerichtet auf Hochleistungssportler gerichtet. 24, 38 Aber Rossetto ${ }^{31}$ präsentiert ein Protokoll zur Verbesserung der sportlichen Leistung im Fußball und Rugby mit den folgenden Punkten: Blase 52 und Blase 58 (Abbildung 8); Blase 17 und Lunge 1 (Abbildung 7).

Der Blasenpunkt 58, strafft die gestreifte Muskulatur des gesamten Körpers, gelenke der Knie, Knöchel und Füße. Es ist ein wichtiger Punkt, um Müdigkeit zu vermeiden, vor allem, wenn mit Magen 36 verwendet. Blase 17 Töne die Dorsalmuskeln, Gelenkbänder und sogar erhöht die Flexibilität der Wirbelgelenke selbst, Förderung regulatorischer Maßnahmen auf das Zwerchfell, Atemrhythmus und Phrenanik. Blase 52 Töne die gesamte Region der Lendenmuskeln und fördert Willenskraft. Lunge 1 wird verwendet, um die Atemarbeit effizient zu machen und ist mit supklavischen und interkostalen Nerven (mit tieferem Einsetzen) verbunden, die im Brachialplexus helfen. Die Verwendung mit Blasenpunkt 17 reguliert den Atemrhythmus.

Wie Bopp Limoge 39, angewendet ein Drei-Punkt-Festprotokoll: Leber 5 (Abbildung 10), Dreifachheizung 15 (Abbildung 7) und Blase 58 (Abbildung 8), zu zwei anderen zufälligen Punkten hinzugefügt, Placebo, bei 35 Athleten, eine signifikante Verbesserung der sportlichen Leistung der getesteten Personen.

Abbildung 10: Leber 5 - 5 tsun über medialen Maleolus; am posteromedialen Rand der Tibia.

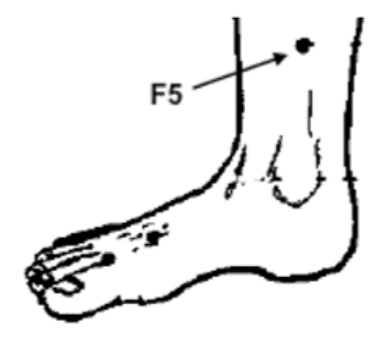

Quelle: Wen ${ }^{17}$ 
Die Studie von Akimot $^{\circ} 38$ bestätigt die Ergebnisse mehrerer Studien zur Verbesserung der körperlichen und geistigen Leistungsfähigkeit, in denen er 21 Fußballsportler während der Wettkampfphase testete, biochemisch die Bewertung von Speicheldrüsen-IgA und Cortisol nach Rate Muskelverspannungen und Müdigkeit, was zu erhöhtem Cortisol und unteren Speicheldrüsen IgA, bestätigt die effektive Verbesserung der sportlichen und geistigen Leistungsfähigkeit.

In einer anderen Forschung in der Behandlung von Sportverletzungen mit kinetischen Akupunktur (Assoziation der Akupunktur mit Kinesiotherapie), in Athleten des Tennis, Fußball, Volleyball, olympische Gymnastik, Capoeira, Ballen und Jiu-jitsu; Patienten mit bewegungsmotorischen Störungen wie: Achillessehnenentzündung, Rückenschmerzen, Rückenschmerzen, Prarkistokus, Tenosynovitis, Karpaltunnelsyndrom, Pronador-Syndrom, Muskelkontusion, Tennis-Ellbogen und Gonalgie aufgrund von Meniskusverletzungen. Alle 31 getesteten Athleten kehrten höchstens bis zur dritten Session ins Training zurück: 53\% kehrten nach der ersten Session zurück; 37\% nach der zweiten; 11\% nach dem dritten, schlussfolgerungsweise, dass diese Technik effizient wirkte, um die Erholungszeit der Athleten zu beschleunigen. Die Erklärung dafür ist, dass Akupunktur in der Hemmung des Krampfzyklus wirkt - Schmerzen, die zu einem segmentalen affektiven Block, supraspinalen absteigenden Block, durch pyramidale Wege und Aktivierung des Prozesses endogene Analgetika führen. ${ }^{26}$

In einer anderen Studie wurden die Variablen maximaler Festigkeit, Explosivkraft, anaerobe Beständigkeit und Geschwindigkeit in hochertragreichen Sprinterkorridoren während einer Übergangszeit bewertet. Die Leber Meridian war fokussiert: Leber 1; Leber 3; Leber 8 (Abbildung 11A), weil die Energiefunktionen dieses Organs sind, um Blut zu speichern, Dispersion zu kontrollieren, Drainage und Sehnen- und Bändererkrankungen zu bestimmen, zusätzlich zur Kontrolle des emotionalen Teils, weil ein Energiedefizit auslösen kann: Depression; Reizbarkeit; Schlaflosigkeit; störende Träume. Der erste punktierte Akupunkt war Blase 62, Gallenblase 34, Niere 3 (Abbildung 11B), gefolgt von der Bauchspeicheldrüsenmilz 3 (Abbildung 1A), Leber 1, Leber 3, Leber 8 (Abbildung 11A), Magen 36 (Abbildung 1B) und Schließen mit dem 
Dünndarm 3 (Abbildung 3B). Leberstiche wurden in der achten Sitzung eingeführt. Die Ergebnisse waren positiv positiv und entwickelten eine Verbesserung der untersuchten Funktionen. ${ }^{24}$

Abbildung 11 A: Leber 1 - 0,1 Tsun über dem seitlichen Winkel des Nagelbettes der Zehen und Leber 3 - zwischen dem ersten und zweiten Metatarsals, hinter den metatarsal-phalangealen Gelenken; Leber 8 - am Ende der medialen Seite der poplitealen Falte, am antero-medialen Rand der semimembraösen und semitendinösen Muskeln. B: Niere 3 - zwischen dem hinteren Rand des medialen Maléolus und der Achillessehne; Gallenblase 34 - I Tsun unter dem Knie, in vorderer und niedriger erbärmlichen Kopf der Fibel, in der Faszie des langen Peroreanmuskels; Bexia 62 - 0,5 tsun unter dem äußeren Maleolus, in der unteren Depression des Maleolus.

Quelle: Wen 17

Ehrlich 25 hing egen teilte 36 männliche Individuen in drei Gruppen ein: eine, die mit systemischer Akupunktur behandelt wurde; eine andere Kontrollgruppe; eine andere mit Placebo-Akupunktur. Eine signifikante Verbesserung der körperlichen Leistungsfähigkeit und hämodynamischen Parameter wurde erreicht. Eine Sitzung fand pro Woche im gubernatorialen Vaso 20, Vaso-Empfängnis 15 und Leber 13, Blase 43 (Abbildung 12), Magen 36 (Abbildung 1B) und Milz-Pankreas 6 (Abbildung 1A) für 5 Wochen statt. Sie analysierten, dass sich die anaerobe Schwelle um 6,62\% verbesserte und die maximale Leistung von 7,15\% in der Gruppe, die systemische Akupunktur erhielt, bereits in der Kontrollgruppe und Placebo, gab es keine Veränderungen. 
Abbildung 12: Governor Vase 20 - auf der Mitte-vertikale Kopflinie; 7 Tsun über dem hinteren Rand des Haares; 5 Tsun hinter dem vorderen Haarrand; vaso Konzeption 15 - 3 Tsun über Zhongwan (RM12), in der Mittellinie des Bauches und der Leber 13 am unteren Rand des Endpunkts der elften Rippe, an der Seite des Bauches; Blase 43 - 3 Tsun, seitlich der Achse (Du-Mai), am unteren Rand der Wirbelsäule (T5).

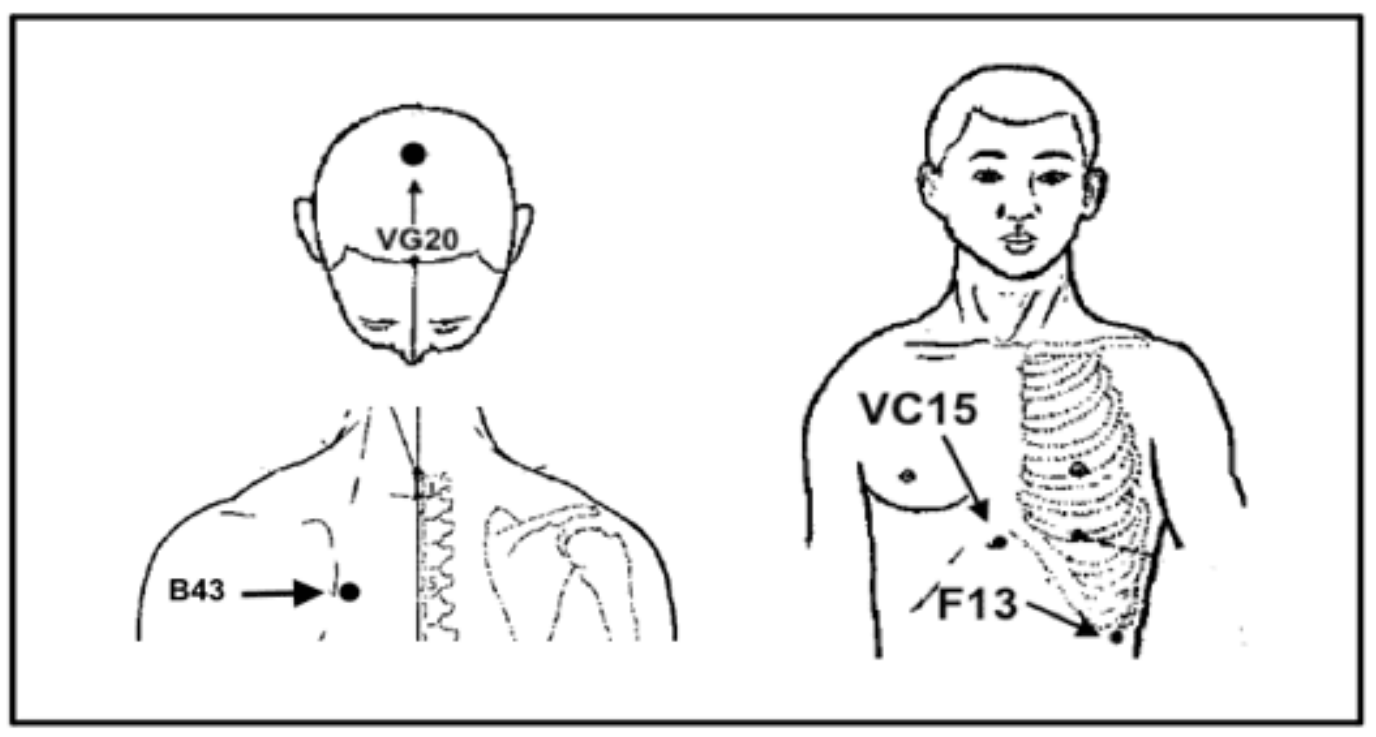

Quelle: Wen ${ }^{17}$

Lin ${ }^{4} 0$, bewertete die Auswirkungen der Ohrakupunktur auf die Herzfrequenz (Cmf), Densauerstoffverbrauch (VO2max) und Blutmilchsäure bei Elite-Basketballsportlern. Sie wurden in 2 Gruppen mit je 12 Athleten eingeteilt, wobei: Gruppe 1 - auricular Akupunktur; Gruppe 2 - Steuerung. Jeder der Athleten radelte auf einem Heimtrainer bis zur Erschöpfung, und die Cmax, VO²max und Blutmilchsäure wurden während der Ruhezeit, nach dem Erhitzen und während des Trainings gemessen: nach 5, 30 und 60 Minuten. Die Ergebnisse zeigten, dass HR und Blutmilchsäure nach 30 und 60 Minuten Aktivität deutlich niedriger waren als in der Kontrollgruppe.

Derselbe Autor, Lin ${ }^{4} 1$, evaluierte in einer neuen Studie die gleichen Variablen wie FCmx, VO2max und Blutmilchsäure in 30 Basketball-Athleten, aufgeteilt in 3 Gruppen: 1- systemische Akupunktur; 2- Scheingruppe; 3-Steuerung. In Gruppe 1 wurden die Durchblutung und Sexualität Punkt 6 (Abbildung 13), Magen 36 (Abbildung 1B) verwendet und während der Studie führte jeder Athlet die Tests auf einem 
Übungslaufband für 15 Minuten durch. Die Variablen wurden in Ruhe und in 5, 30 und 60 Minuten nach dem Training gemessen, was eine signifikante Reduktion der drei Variablen nach 30 und 60 Minuten erhielt.

Abbildung 13: Durchblutung und Sexualität 6 - 1 Tsun unter Jianshi-Punkt (PC5); 2 Tsun über dem Handgelenk, zwischen Sehnen langer Palmarmuskulatur und karpalradialem Flexor

Quelle: Wen 17

Dhillon 42, bewertete die Leistung von 20 männlichen Radfahrern, unterteilt in drei Gruppen: behandelt mit systemischer Akupunktur: Magen 36 (Abbildung 1B), Gallenblase 34 (Abbildung 11B), Leber 11 (Abbildung 14), Niere 3 (Abbildung 11B), Regler 20 (Abbildung 12 ); Scheinakupunktur; Kontrolle, ohne Intervention. Die Athleten radelten mit dem stationären Fahrrad $20 \mathrm{~km}$ lang mit maximaler unterstützter Geschwindigkeit. Die analoge Skala des Schmerzes, visuell, für die unteren Gliedmaßen, induziert durch Bewegung; Borgs Ausmaß der wahrgenommenen Anstrengungen; Blutlaktatkonzentrationen nach der Übung. Die akupunkturinduzierte Gruppe präsentierte eine höhere Punktzahl in der Borg-Skala, was bedeutet, dass die Behandlung eine größere Anstrengung während der Aktivität förderte und die Zeit nach Abschluss des Tests verringerte.

Abbildung 14: Leber 11 - 1 Tsun unten ingüinal; an der antero-medialen Kante der Schwelle des langen Adduktorenmuskels

Quelle: Wen 17

Laut Hubscher 43 evaluierte er den maximalen Sprung mit bipodaler Landung und freiwilliger maximaler isometrischer Stärke der Quadrizeps, mit Oberflächenelektromyographie, um die Femoral-Rectusmuskelaktivität für 30 Sekunden der Unterstützung zu messen. Dreiunddreißig Athleten wurden (13 weiblich und 20 männlich) in drei Gruppen eingeteilt: systemische Akupunktur mit Magenpunkten 36 (Abbildung 1B), Milz-Bauchspeicheldrüse 6 (Abbildung 1A), VasoKonzeption 6 (Abbildung 9); Akupunkturgruppe mit nicht vorhandenen Punkten; 
Kontrollgruppe verwendet Laser deaktiviert. Als Ergebnis gefunden, es war eine nicht signifikante Erhöhung des maximalen Sprungs, aber eine wichtige Erhöhung der isometrischen Stärke der Quadrizeps.

In einer anderen Geng 44-Umfrage rekrutierte er 12 gesunde Männer mit Sportstudenten zwischen 19 und 25 Jahren, die die Auswirkungen der Ohrakupunktur auf den Milchsäurespiegel im Blut bewerteten, die durch Bewegung auf das Laufband (VO2max) induziert wurden. Er wählte die Punkte: Leber, Lunge, Subcortex, Endokrine Drüsen und Dreifachheizung (Abbildung 15). Die Ergebnisse waren eine Erhöhung der Sauerstoffkapazität Gebäude und verringerte Blut Milchsäurespiegel nach Übungen.

Abbildung 15: Ohrpunkte

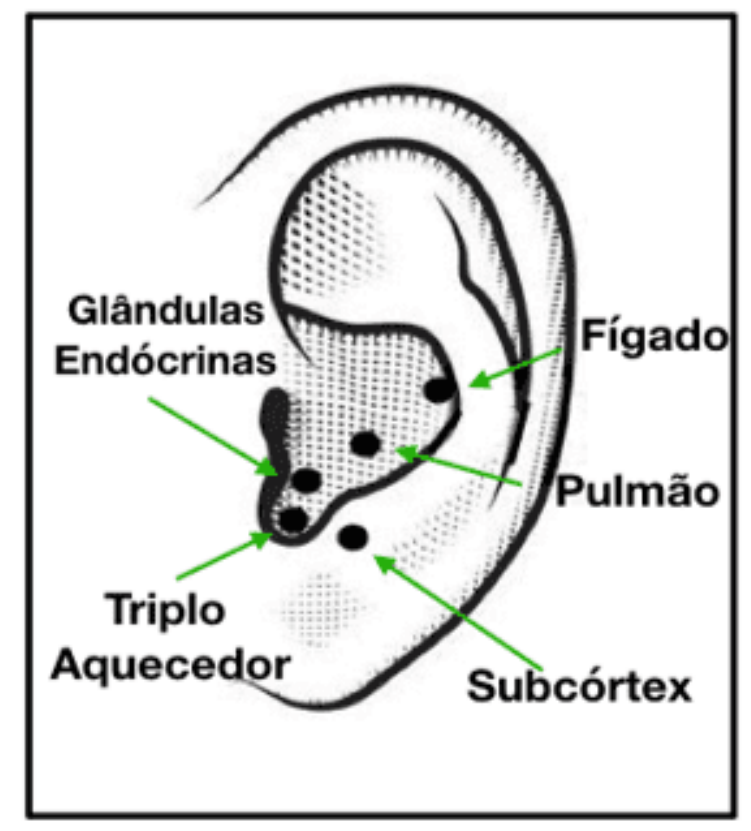

Quelle: Personal Archiv des Autors

Eine weitere Studie von 28 männlichen Individuen in eine Kontroll- und Experimentalgruppe unterteilt. Eine Datenerfassung wurde vor Akupunktursitzungen durchgeführt: Messung der Basalherzfrequenz durch das Oximeter; Herzfrequenzmessung nach physikalischen Tests in der Harvard Bank, mit einer Höhe von $50 \mathrm{~cm}$, Durchführung von Auf- und Abstiegen für 5 Minuten; Herzfrequenz 
gemessen in eineinhalb Minuten des Tests, 2 und eine halbe Minute und dreieinhalb Minuten; Berechnung des Physical Fitness Index (IAF); durchschnittliche Herzfrequenz und Anzahl der Aufstiege und Abstiege während harvard Bank Tests. Die Punkte wurden verwendet: Lunge 10 und Vaso-Empfängnis 17 (Abbildung 16), Dünndarm 3 (Abbildung 3B), um zu straffen und zu Muskelentspannung, Stärkung sehnen, Lendenwirbelsäule und untere Gliedmaßen führen. In den Gallenblasenpunkten 34 (Abbildung 11B) standen Milzbauchspeichelspeicheldrüse 4 und Niere 7 (Abbildung 16) im Zusammenhang mit der Sedierung, um die Bewegung der Durchblutung (Xue) und Lymphe (Jing Yie) zu potenzieren. Acht Sitzungen wurden durchgeführt, eine pro Woche und die Ergebnisse zeigten keine signifikante Verbesserung in Bezug auf Herzfrequenz und körperliche Fitness. Bei der Steigerung der körperlichen Leistungsfähigkeit, Steigungen und Abstiegen von der Harvard Bank waren die Werte für die experimentelle Gruppe in Bezug auf die Kontrolle signifikant. 36

Abbildung 16: Lunge 10 - auf der Palmarseite, über dem Gelenk des ersten digitalen Metakarpalen, zwischen dunklen und hellen Fellen; Vasenkonzeption 17 - in der medianen Linie des Brustbeins auf höhe der Brustwarze; Niere 7 - 2 Tsun über Taixi (R3); an der antero-medialen Kante des Seemuskels; Milz Bauchspeicheldrüse 4 - auf der medialen Seite des Fußes; 1 Tsun hinter dem metatarsophalen Gelenk, an der Kreuzung von dunkler und heller Haut.

Quelle: Wen 17

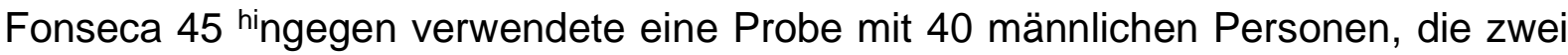
Gruppen an Muskeldehnung übten: Kontrolle und Intervention, um die Auswirkungen der Akupunktur auf die lokalisierte Muskelausdauer (RML) der oberen Gliedmaßen zu bewerten. Die Kontrollgruppe wurde dem RML-Test für 1 Minute unterzogen, während einer Ruhezeit von 30 Minuten und nach dem Test wurde der Test wiederholt.

In der Interventionsgruppe wurde der Test angewendet: der Test; 5 Minuten ausgeruht; Anwendung der Akupunktur, Toning der Punkte: Milz Bauchspeicheldrüse 3 (Abbildung 1A); Niere 7 (Abbildung 16); Magen 36 (Abbildung 1B); Gouverneursvase 
4; Dreifachheizung 15 (Abbildung 7); für 25 Minuten. Nach der Anwendbarkeit wiederholte sich der RML-Test, was zu einer signifikanten Verbesserung der Muskelleistung der Interventionsgruppe führte.

Im Jahr 2015, Moniz, verwendet 12 Rugby-Athleten, wo sie in Kontrollgruppe und Testgruppe verteilt wurden, Durchführung Akupunktur für 20 Minuten in den Punkten: Herz 5, Blase 15, Blase 44 (Abbildung 17) und Kreislauf und Sexualität 6 (Abbildung 13). In der zweiten wöchentlichen Sitzung wurden Punktionen an den Spannungspunkten und Auslösepunkten durchgeführt. Die Ergebnisse legten eindeutig nahe, dass die Anwendung der Akupunktur bringt Vorteile für Die Athleten über Muskelprobleme, mit totaler Erholung auf Schmerzniveau und Begrenzung der Bewegungen vor dem anschließenden Spiel die Verletzung.

Abbildung 17: Herz 5 - auf der ventralen und ulnaren Seite des Unterarms, auf der ulnaren Seite der Sehne des Karpal-Ulnar-Flexormuskels; 1 Tsun über dem Griff; Blase 15 - am unteren Rand des Wirbelsäulenprozesses des Wirbels (T5), auf der gleichen vertikalen Linie wie der Feishu-Punkt (B13); Blase 44 - 3 Tsun, seitlich der Achse, am unteren Rand der Wirbelsäule (T6).

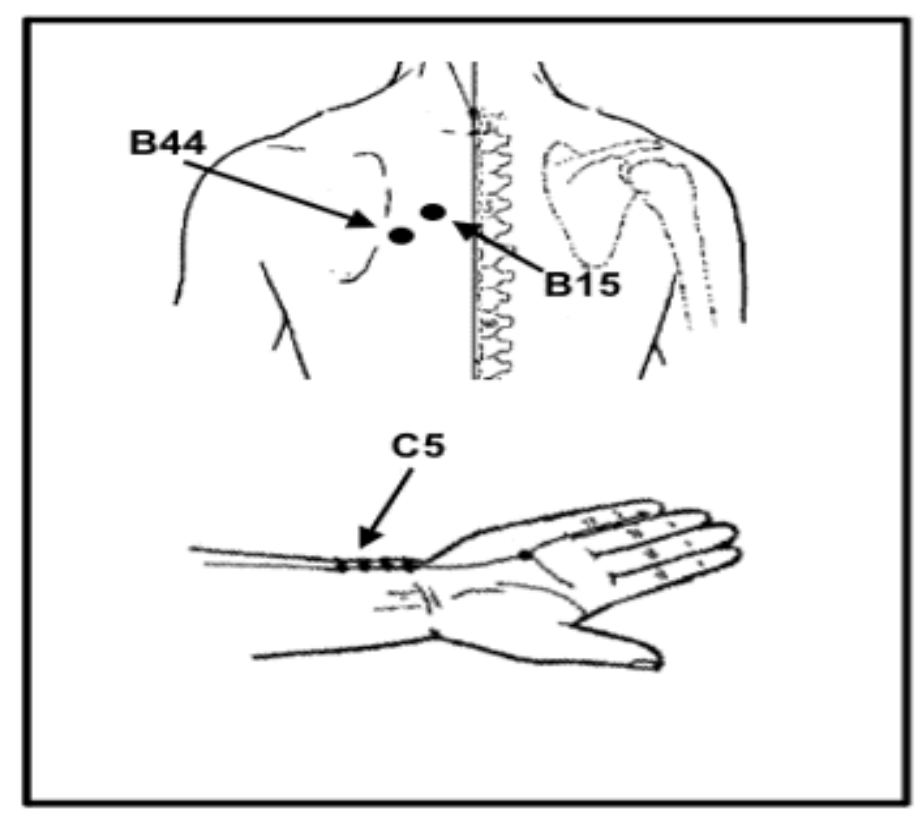

Quelle: Wen ${ }^{17}$ 
Die meisten Studien liefern signifikante Ergebnisse zur Verbesserung der körperlichen Leistungsfähigkeit, was zu einer Verbesserung der Zeit nach dem Ende der Tests führt. Dies bewirkt, dass Personen, die einer Akupunkturbehandlung unterzogen werden, eine Aktivierung der Neurophysiologie der Schmerzkontrolle erhalten, was eine Verbesserung der Muskelleistung auslöst und das Endergebnis stark beeinflusst. 46

\section{ACUPUNCTURE USED IN DER BEHANDLUNG VON LESIONS}

Akupunktur wirkt in diesem Prozess der Heilung der Krankheit, Beseitigung ihrer Ursache, und wird daher sowohl für die Behandlung von verschiedenen akuten und chronischen Schmerzen verabreicht. Es kann in chirurgischen Verfahren, bei der Verbesserung der körperlichen Bedingungen und bei der Prävention von Verletzungen in verschiedenen Sportarten verwendet werden. ${ }^{24}$

Derzeit suchen Athleten und ihre Trainer die Verbesserung der körperlichen Leistungsfähigkeit und Prävention durch umfassendes Training, Ernährungsüberwachung, Physiotherapeupic-Prävention, Trainingspläne, psychologische Überwachung und Verwendung von neuen Sportgeräte, die Ziele erreichen. Auch mit diesen verbesserten Systemen, viele Athleten bevorzugen und bewegen sich auf dem schnellsten Weg, mit illegaler Behandlung, wie die Verwendung von Anabolika und Drogen. ${ }^{32}$ Nach Frasca 35, führt er aus, dass Akupunktur im Menschen als Ganzes wirkt, Schmerzen behandelt, die Leistung verbessert und direkt im psychologischen System der Athleten wirkt, was zu einer Erhöhung des psychischen Vertrauens des Sportlers führt.

Laut Santos und Mitarbeitern ${ }^{20}$, die meisten Athleten haben die Erfahrung des Gefühls spät einsetzenden Muskelschmerzen, die in Trainingszeiten auftreten, wo die Intensität und das Volumen der Arbeit höher sind. Eine andere Art von Schmerzen wird durch eine Verletzung verursacht, die Athleten für längere Zeit vom Training und Wettkämpfen wegbewegt. ${ }^{21}$ 
Basierend auf neurophysiologie, histoanatomisch und neurochemisch, CTM erklärt, dass durch Punturating der Punkt, der in den Kanälen der spezifischen Meridiane befindet, wird es den Energiefluss in diesen Strukturen durch Sportmodalitäten überlastet erleichtern, analgetische Ziele zu erreichen. ${ }^{25,47}$

Andere Autoren berichten, dass die Anwendung der Technik die Wirkung von endogenen Opioiden hat, die Freisetzung von Endorphin, das in der Schmerzmodulation und Hemmung der nozizeptiven Übertragung auf allen Ebenen des Nervensystems identifiziert wird, erfolgt.) durch die Verwendung von Elektroakupunktur, positive Regulation der Expression des Verhältnisses der neuronalen Stickstoffmonoxidsynthese (nNOS) / NADPH-Diaphorase (NADPHd) tritt auf. Gemessen durch thalamische Bahnen und die dorsale Medulla. Es gibt auch einige Daten, die darauf hindeuten, dass neben Opioiden, andere Mechanismen sind in der Behandlung von Schmerzen beteiligt. ${ }^{6,48,49,50,51}$

Das Vorhandensein von NO begünstigt die Verbesserung der lokalen Vaskularisation, was auf eine homeostotische Wirkung der Akupunktur hindeutet, aber die Zunahme von NO im eisigen Faszikel kann diese regulatorische Wirkung somatischer Reflexe, die durch die Technik verursacht werden, klären. ${ }^{6,52}$ Andere Studien beobachten die Freisetzung von Serotonin nach Nadelstimulation. ${ }^{51,52,53}$

Akupunktur wird angewendet, um Ton, entspannen Athleten Muskeln und halten die Energiebilanz des Körpers. Zusätzlich zur schmerzstillenden Wirkung ist es möglich, andere biologische Faktoren zu stimulieren, indem der Hypothalamus und die Hypophyse systemische Effekte erzielen, wie z. B.: erhöhte Neurohormone und Neurotransmitter.2 Verbesserung der Leistung des Athleten in ihren jeweiligen

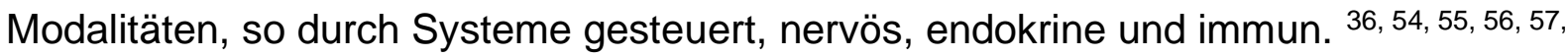
58, 59, 60 Viele Studien haben gezeigt, dass Akupunktur in diesen drei Systemen funktioniert und: Linderung von Spannungen und Muskelschmerzen; erhöhte lokale Durchblutung; Freisetzung von endogenen Opioiden, abnehmende Schmerzwahrnehmung; Verbesserung der Modulation des gleichgewichtenden und parasympathischen Nervensystems; Modulation des Immunsystems; Aktivierung von Bindegewebe und Muskeln Freisetzung von Myosinen, die entzündungshemmende 
Wirkung haben, Freisetzung von antioxidativen Substanzen, Verringerung des oxidativen Stresses; Stoffe, die wahrscheinlich ergogene Wirkungen haben. 5, 13, 61, 62, 63,64

In einer Laborstudie mit Ratten, Lee und Kollaborateuren berich ${ }^{\text {teten }}$ 65, dass das Tier, wenn es akupunkturiert und trainiert wurde, eine Unterdrückung der Wirkungen auf 5 HT (5 Hydroxytryptamine) und die Expression von THP (Hydroxylase Tryptophan) in der dorsalen von Meerschweinchen, die ergogene Wirkung erreichen, da das 5 HT die Durchführung von Bewegung bei Menschen und Ratten verhindert.

In einer Studie berichteten Zylone ${ }^{y}$ und 66 Mitarbeiter, dass es möglich ist, eine andere Aktivität im Gehirn zu finden, durch Magnetresonanzbewertung, während des Nadelverfahrens. Diese Veränderungen treten in Regionen wie: epiaquedurale graue Materie, Hypothalamus, primäre somatosensorische Kortex, oberen zeitlichen Gyrus, rostralan anterior cingulate cortex und mediale Region des okzipitalen Kortex. ${ }^{48}$

In seiner Forschung behan ${ }^{\text {delte }}$ Minori 67 sich wiederholende Schulterstressverletzungen, mit der Kombination von wunderbaren Gefäßstichen: Dreifachheizung 5 - Gallenblase 41 (Abbildung 18), die für chronische Schmerzen und Dünndarm verwendet werden 3 (Abbildung 3B) - Blase 62 ( Abbildung 11B) bei akuten Schmerzen.

Abbildung 18: Dreifachheizung 5 - 2 Sonnen über der Rückenfalte des Handgelenks, zwischen den Sehnen des gemeinsamen digitalen Extensormuskels und dem fünften Stellenmuskel selbst; Gallenblase 41 - in Depression zwischen dem vierten und fünften Metatarsals.

Quelle: Wen 17

Die lokalen Punkte für die Behandlung sind: Dickdarm 5 für Handgelenkschmerzen; Dickdarm 4 lindert Schmerzen und Ödeme (Abbildung 4 A); Dünndarm 9 Armschmerzen; Dreifachheizung 14 für Schmerzen und Entzündungen der Schulter angezeigt; Dickdarm 14 (Abbildung 19) für Steifigkeit, Parese, eingeschränkte Schulterbeweglichkeit. Entfernte Punkte, Ober- und Vorderschulterschmerzen: Magen 
36, um externe pathogene Faktoren zu zerstreuen; Magen 37 indiziert für Schmerzen, Empfindlichkeit und Durchblutungsstörungen; Magen 41 eliminiert Feuchtigkeit, Stagnation, Wind und beruhigt den Shen (Geist); Magen 38 eliminiert akute Schmerzen und Schultergelenkserkrankungen (Abbildung 1B). Bei hinteren und überlegenen Schulterschmerzen: Gallenblase 34 für eingeschränkte Beweglichkeit, Krämpfe, Durchblutungsstörungen und Funktionsstörungen von Muskeln und Sehnen; Gallenblase 38 dispergiert den Wind und gibt Wärme ab, aktiviert den Meridian und seine Gefäße (Abbildung 11B). Schmerzschulterblatt, auf der anderen Seite: Blase 65 pacifis und stärkt den Geist, löst Wärme und lindert Schmerzen; Blase 64 unterdrückt Krämpfe und entspannt Sehnen; Blase 60 lindert Schmerzen und aktiviert die Meridian- und Emotionalpunkte (Abbildung 2A); Blase 18 fördert die allgemeine Ruhe und beseitigt Krämpfe (Abbildung 19) und Leber 8 entspannt Sehnen (Abbildung 11A).

Abbildung 19: Dünndarm 9 - im postterounteren Bereich des Schultergelenks, auf der postteroinferioren Seite des größeren runden Muskels; Dreifachheizung 14 - in Depression zwischen dem achromen und dem größeren Humerus tuberculo, am Rand der Infraspinalsehne; Dickdarm 14 - bedeckt die Seitenseite und einige radiale des Arms, am distalen Punkt des Deltamuskels, 3 Tsun unter jianyu Punkt; Blase 18 - auf der gleichen vertikalen Linie wie geshu (B17), am unteren Rand des Wirbelsäulenprozesses des Wirbels (T9).

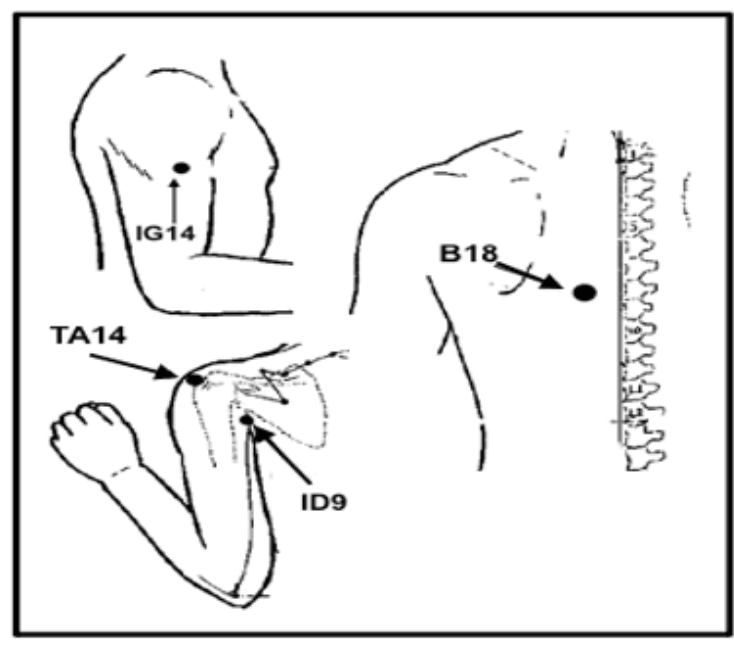

Quelle: Wen ${ }^{17}$ 
Brum 68 hingegen, in seiner Studie zur Behandlung von Pyriform-Syndrom, Massage und Akupunktur-Techniken in Fluren verbunden, um Muskelfunktionen, Flexibilität und Linderung der Symptome zu verbessern. Die Forschung umfasste Athleten beider Leies im Alter von 30 bis 60 Jahren. Die Sitzung konzentrierte sich auf Massage (Shiatsu) zu Beginn, um die Spannungspunkte der Gesäß- und Oberschenkelregion auf der betroffenen Seite zu lösen, gefolgt von Nadeln, wie folgt: im Uhrzeigersinn; Anti-Zeit; Vertiefung; oberflächliche Die Nadel in den folgenden Bereichen: Ursprung und Insertion des piriformen Muskels (Beckengesicht des Kreuzbeins und größerer Trocanter des Oberschenkelknochens); Ursprung des mittleren Gluteus (laterales Gesicht des Iliasknochens); Obergrenze des Mindestgluteus; Obergrenze des Gluteus-Maximums; Ursprung der Hamstring-Muskeln (Ischiastik-Tubrosität); femorale Bizeps Muskel und semitendinöse Muskeldiaphyse, mit Massage beendet.

Die Ergebnisse zeigten eine signifikante Verbesserung, wenn im Zusammenhang mit der Verbesserung der Schmerzen, Flexibilität, Schmerzpalpation im piriformen Muskel und Test für Pyriform-Syndrom. Dies zeigt die Übereinstimmung mit der Studie von Ho ${ }^{\text {ng }}$ wen 69, wo die Wirksamkeit der Akupunktur 97,33\% betrug und mit der Forschung von France 26, die die Nadeln mit Kinesiotherapie assoziierte, die eine Linderung der Symptome, die Potentialisierung des neuromuskulären Systems, eine schnellere Rückkehr zu sportlichen Aktivitäten.

In seiner Arbeit, Rocha ${ }^{70}$, überprüfte die Verbesserung der Aktivierung des femoralen Rectusmuskels, durch Elektromyographie, nach Stimulation des Magenakupunkts Punkt 45 (Abbildung 20). Insgesamt wurden 10 Teilnehmer (5 Männchen und 5 Weibchen) ausgewählt, wo sie eine Übung von 2 Kniebeugen-Wiederholungen durchführten, wo sie den höchsten Muskelaktivierungsindex unter den beiden Bewegungen durch das elektromyographische Signal wählten. Dann wurde Akupunktur aufgetragen, den Magenpunkt 45 für 10 Minuten getopfet und dann wurde die isometrische Kniebeuge in der unteren Phase wiederholt. Nach den Ergebnissen gefunden Akupunktur veränderte die elektrische Aktivität des femoralen Rectusmuskel während der isometrischen Phase in der unteren Phase der Kniebeuge, als wirksam. 
Abbildung 20: Magen 45 - 0,1 Tsun an der Seite der Seitenecke des Nagelbettes der zweiten Zeh

Quelle: Wen 17

In einer anderen Studie Pinheiro 71 , charakterisiert und legte einige Punkte für die Behandlung der Meniskalläsion: Leber 8 (Abbildung 11A) verwendet, um den Leberkanal mit der Funktion der Harmonisierung und Straffung Leber und Blut Qi, Förderung der Fülle der Nieren und zur Entspannung von Sehnen und Muskeln führen, stärkung den lq des Knies. Der Blasenpunkt 40 (Abbildung 5) findet sich in der poplitealen Fossa, die es ermöglicht: Dispersion von Wärme und perversem Wind der Gliedmaßen; Entspannung der Muskeln und Sehnen; Zerstörung von Blutgefäßen; reduziert Die Hitze und eliminiert die Blutstase; verringert regidez und stärkt knie- und lenden. Auf der anderen Seite mobilisiert der Nierenpunkt 10 (Abbildung 21) Wasser; Töne yin Qi; vertreibt Feuchtigkeit und perverse Hitze, Behandlung von Knieschmerzen, Beinödem und betäubenden Knie- und Poplitealenenzeszen.

Abbildung 21: Niere 10 - auf der medialen Seite der poplitealen Falte, zwischen semitendinösen und semimembraösen Muskeln

Quelle: Wen 17

In einer Studie über Schulterfunktionsstörungen, N ${ }^{u n}$ es 72, überprüfte die Bibliographie und legte die Punkte der wunderbaren Gefäße: Dünndarm 3 (Abbildung 3B) - Blase 62 (Abbildung 11B) um Funktionen auszugleichen, entspannende Muskeln und Sehnen; die Punkte der Dreifachheizung 5 - Gallenblase 41 (Abbildung 18) lindern Schmerzen und entspannen Sehnen; Ashi-Punkte; Leber 8 (Abbildung 11A) und Niere 7 (Abbildung 16) zur Stärkung der Sehnen; Magen 36 (Abbildung 1B) Beseitigung von Kälte und Feuchtigkeit aus den Gelenken; Darm 4 (Abbildung 4A) und Dickdarm 15 (Abbildung 22) wirkend in Bewegung und Schmerz der Schulterflexion; Dreifachheizung 14 (Abbildung 19) und Dickdarm 16 (Abbildung 22) zur Verbesserung der Erweiterungsmobilität und -schmerzen. 
Abbildung 22: Dickdarm 15 - auf der Oberseite der Schulter, am seitlichen Rand des Akromons gibt es zwei Depressionen, dieser Punkt ist in der vorderen Depression; Dickdarm 16 - in Depression zwischen dem oberen und hinteren Rand des akromioklavikulären Gelenks und der Wirbelsäule des Schulterblattes.

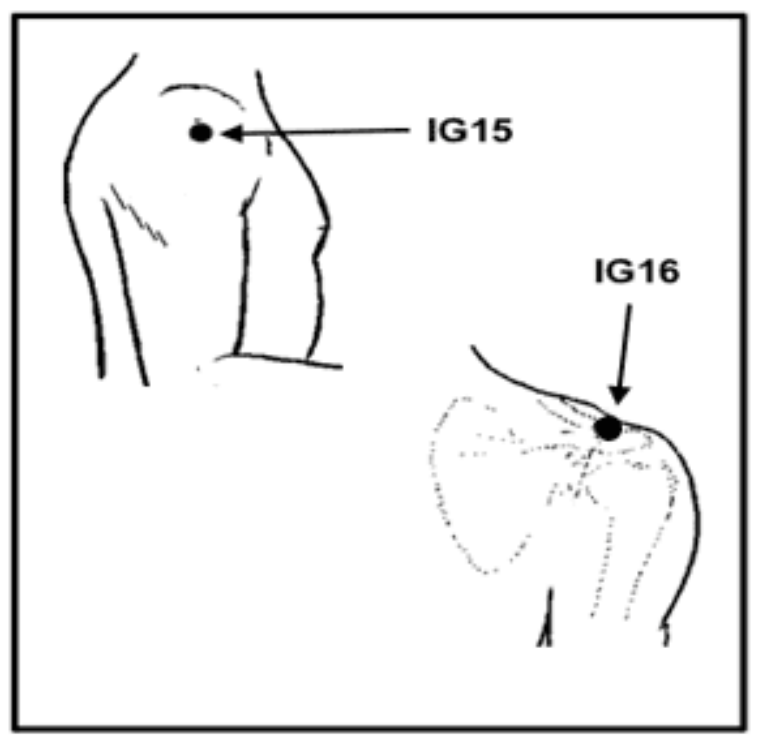

Quelle: Wen ${ }^{1}$

Um die unmittelbaren Auswirkungen der Akupunktur auf spät auftretende Muskelschmerzen (DMIT) und kontraktile Funktionsfähigkeit der Muskeln zu überprüfen, wählte Parisoto 73 , ausgewählte 30 Probanden (12 Männer und 18 Frauen), im Alter zwischen 20 und 30 Jahren, Praktiker der 2 bis 3 Mal pro Woche. Sie wurden in drei Gruppen eingeteilt: mit Akupunktur behandelt; Placebo-Schein; Steuerung. Folgende Werte wurden bewertet: Schmerzschwelle mit dem Algelometer; maximale isometrische Muskelkraft; elektromyographische Aktivität; subjektive Schmerzwahrnehmung durch die visuelle analoge Skala.

Nach den Auswertungen wurden die Probanden der Induktion von DMIT unterzogen, wobei die Flexion und Verlängerung des Ellenbogens mit Last (Bizepsfaden) bis zur maximalen Grenze der Bewegungsausführung durchgeführt wurden: 3 Serien wurden bis zur Muskelerschöpfung mit einem Intervall von 4 bis 5 Minuten durchgeführt. Nach 
diesem Eingriff wurde der Zustand, in dem sich der Muskel vor der Akupunktur befand, neu bewertet.

Die für die Behandlung der Akupunkturgruppe ausgewählten Punkte waren: Dickdarm 4 (Abbildung 4A), Dickdarm 11 (Abbildung 4B), Magen 36 (Abbildung 1B), Gallenblase 34 (Abbildung 11B), die schmerzstillenden und tonenden Punkte von Muskeln und Sehnen sind. In der Scheingruppe wurden Placebopunkte verwendet und die Nadeln oberflächlich eingeführt; Kontrollgruppe legen die Personen einfach fest. Beide Gruppen blieben 20 Minuten in Ruhe.

Die Ergebnisse zeigten: Die Schmerzwahrnehmung mit der analogen Skala wurde in der Akupunkturgruppe reduziert; die Schmerzschwelle des Algelometers erhöhte sich nach Intervention in dieser Gruppe. Nach akupunktionischer Intervention wurde die Bizepsmuskelaktivierung um $30 \%$ reduziert, wenn sie mit der Schein- und Kontrollgruppe in Verbindung gebracht wurde. Die Muskelkraft verringerte sich nach DMIT in der getesteten Gruppe um $48 \%$ und war damit niedriger als in den Scheinund Kontrollgruppen (58 \% bzw. 56 \%). Alle Gruppen präsentierten maximale sittäre Muskelkraft Defizit nach dem Dmit-Induktionsprotokoll: Akupunktur verringerte sich um $26 \%$; Schein $29 \%$; $33,5 \%$.

Es wurde jedoch festgestellt, dass Akupunktur bei der sofortigen Verbesserung der Schmerzwahrnehmung und erhöhter Schwelle durch Algelomer wirksam war, was neuromuskuläre Veränderungen zur Verfügung stellte, aber nicht die kontraktile Leistung der Muskulatur verbesserte.

In einer anderen Tolentin-Studie ${ }^{74}$ untersuchte sie die Wirkung der Auriculotherapie auf Schmerzen, Funktionalität und Beweglichkeit von Personen mit chronischen Rückenschmerzen. Aufgeteilt 31 Teilnehmer in drei Gruppen: Experimentemit einwegrende Edelstahl-Blutnadeln von $1,5 \mathrm{~mm}$; experimentell mit Senfsamen; Kontrolle ohne Eingriff. Die visuelle analoge Skala wurde verwendet, um Schmerzen zu bewerten; für den Funktionstest wurden der Québec-Fragebogen für Die Lendenwirbelsäule und die Prüfung zur Dauerprüfung verwendet; Lendenwirbelsäulenmobilität durchgeführt. 
Die Interventionen wurden für 4 Wochen durchgeführt, als eine wöchentliche Sitzung, angewendet in den Punkten: Shenmen, Rim, Sympathetic, Analgesie, Muskelentspannung, Lumbar und Nebennierenwirbelsäule (Abbildung 10). Nach einem Intervall von 1 Woche nach der letzten Anwendung wurden die Personen mit dem gleichen Protokoll neu bewertet.

Die Ergebnisse zeigten eine Verbesserung des Zustands von Rückenschmerzen sowie die Verbesserung der funktionellen Kapazität in beiden experimentellen Gruppen, im Gegensatz zu der Kontrolle, dass es keinen signifikanten Unterschied in Bezug auf anfängliche Schmerzen gab. Es wurde kein Unterschied zwischen den durchgeführten Techniken (Nadel und Samen) gefunden, was darauf hindeutet, dass beide vorteilhaft für die Linderung von chronischen Rückenschmerzen und erhöhte Funktionsfähigkeit sind.

Abbildung 23: Ohrpunkte

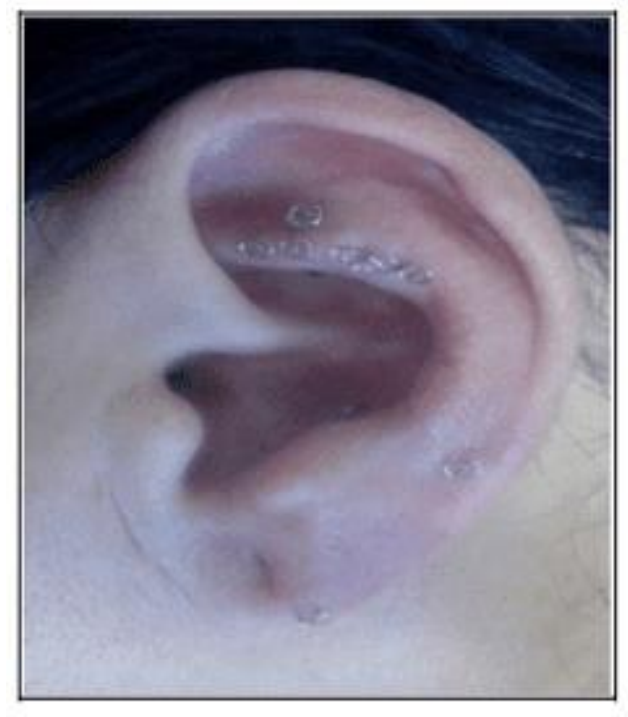

Quelle: Tolentino ${ }^{74}$

\section{ABSCHLIEßENDE ÜBERLEGUNGEN}

Basierend auf der Literatur, die Analyse aller Ergebnisse und ihre wissenschaftlichen Beweise, Es wird angenommen, dass Akupunktur Behandlung wirksam bei der 
Behandlung, Verbesserung der Leistung und Prävention von Verletzungen bei Profiund Amateursportler. Personen, die die Intervention erhalten, auch wenn sie eine Art von verletzten Komplikation haben, ist das Problem weniger schwer und schnell wiederherzustellen organische Funktion, das heißt, der Athlet, der eine Art von Verletzung erleidet und Akupunktur durchführt, diese Widrigkeiten werden in der Regel minimale und frühe Rückkehr Aktivitäten sein.

Auch Athleten, die akupunktur nicht verwenden, um Prävention zu verhindern und präsentieren die Läsion, die Intervention dieser präsentiert klinische Beweise, basierend auf Neurophysiologie, Opioid-Freisetzung, mit Schwerpunkt auf der Behandlung von Schmerzen und Verbesserung des Wohlbefindens des Patienten.

Akupunktur Intervention, obwohl es eine tausendjährige Technik ist, wächst immer noch im Westen, hat große Ergebnisse in der klinischen Praxis, Fragen der Prävention von Muskel-Skelett-Verletzungen, und auch in der Prävention und Behandlung von Krankheiten psychosoziale, akute und chronische Schmerzen.

Aufgrund des Gleichgewichts des Energiesystems behandelt Akupunktur nicht nur das körperliche Wohlbefinden, sondern auch das geistige Wohlbefinden, da die theoretische Grundlage der Technik den Körper nicht vom Geist trennt, der emotionalen Stress begünstigt, Angst reduziert und die menschlichen Geist.

\section{VERWEISE}

1. Ching N. O livro de ouro da medicina chinesa. 3. ed. Rio de Janeiro: Objetiva; 1991.

2. Malveira CL, Souza FCS, Rocha TBX. Biomecânica do movimento isométrico de pedala antes e após aplicação de acupuntura: Um estudo preliminar. Motric. 2012 Set;8(52):630-9.

3. Sousa NR. Relação entre a reatividade de pontos de acupuntura e atividade física com afecções ortopédicas determinadas por exames de imagens em equinos. Botucatu. Tese [Doutorado em Biotecnologia Animal] Universidade Estadual Paulista; 2015. 
4. Silveira NC, Martins RCC. Eficácia da acupuntura para o tratamento da fibromialgia: Uma revisão sistemática. Psicol e Saúde em Debate. 2018 Fev; 4(1):85-105.

5. Sant' Anna FM, Rangel VM, Mota DDS. Tratamento da Tenossinovite de De Quervain por Acupuntura - Relato de Caso. Vittalle. 2018 Mar; 30(1):130-6.

6. Tsuchiya M, Sato EF, Inoue M, Asada A. Acupuncture enhances generation of nitric oxide and increases local circulation. Anesth Analg. 2007 Feb; 104(2):3017.

7. Tavares MG, Machado AP, Motta BG, Borsatto MC, Rosa AL, Xavier SP. Electro-acupuncture efficacy on pain control after mandibular third molar sugery. Brz Dent J. 2007; 18(2):158-62

8. Hutchinson AJ, Ball S, Andrews JC, Jones GG. The effectiveness of acupuncture in theating chronic non-specific low back pain: a systematic review of the literature. J Otthop Surg Res. 2012 Oct 30;7:36.

9. Moniz PJL. Avaliação da acupuntura no desempenho do rugby. Coimbra. Dissertação [Mestrado Integrado em Medicina] - Faculdade de Medicina da Universidade de Coimbra; 2015.

10. Magalhães PC. Efeito da acupuntura na prevenção da hemorragia pulmonar induzida por exercício em cavalos puro sangue inglês de corrida. Botucatu. Dissertação [Mestrado em Biotecnologia Animal] - Universidade Estadual Paulista; 2016.

11. Matias $B$ et al. Acupuntura de equilíbrio como coadjuvante no tratamento de disfunções temporomandibulares. Ação Odonto. 2018 Jun; 1(1):65-72.

12. Franco A. Acupuntura Ajuda Atletas nas Lesões e na Performance. Jornal da Madeira 2016 agosto 2;p.25.

13. Lopes SS, Mota MPG. Efeito da acupuntura na resistência física após exercícios repetitivos de tornozelo - Estudo experimental. R bras Ci e Mov. 2018 Jul;26(1):13-21.

14. Lopes SS, Mota MPG. Influência da acupuntura no limiar de percepção dolorosa de musculatura submetida a esforço repetitivo. Br J Pain. 2018 Jul; 1(3):207-11. 
15. Cohen M, Abdala RJ, Ejnisman B, Amaro JT. Lesões Ortopédicas no futebol, Rev. Bras. Ortop 1997 Dez;32(12):940-44.

16. Dias Junior JC, Marino DM. Acupuntura na prevenção de lesões musculares em atletas de futebol profissional. Rev Fisioter S Fun. Jan-Jul:6(1):36-42.

17. Wen TS. Acupuntura Clássica Chinesa. 15. ed. São Paulo: Cultrix, 2006.

18. Maciocia G. Os fundamentos da medicina chinesa: um texto abrangente para acupunturistas e fitoterapeutas. São Paulo: Roca; 1996.

19. Ross J. Combinações dos Pontos de Acupuntura: A Chave para o Êxito Clínico. 1 ed. São Paulo: Roca, 2003.

20. Santos VC, Kawano MM, Banja RA. Acupuntura na melhora da performance em atletas juvenis de handebol, Rev Saúde e Pesq 2008 Set-Dez;1(3):331-35.

21. Rubio K, Godoy Moreira F. A dor em corredores com fascite plantar: o uso da acupuntura, Rev Dor 2008 Jul-Set;9(3):1290-1296.

22. Pires TF, Pellegrinotti IL. Acupuntura na Performance Atlética: Estudo Exploratório. [8 ${ }^{a}$ Mostra Acadêmica da UNIMEP; 2010 out; 1-4; Piracicaba, Brasil].

23. Yang HY, Liu TY, Gao M. Electrical acupoint stimulation increases athletes rapid strength, Zhongguo Zhen Jiu 2006 May;26(5): 313-315.

24. Luna MP, Fernandes Filho J. Efeitos da Acupuntura na performance de Atletas velocista de alto rendimento do Rio de Janeiro. Fit e Perform J 2005 Julh/Ago;4(4):199-214.

25. Ehrlich D, Haber $P$. Influence of acupuncture on pysical performance capacity and Haemodynamic Parameters. J. Sports Med 1992 May;13(6): 486-91.

26. França D, Fernandes-Senna V, Cortez CM. Acupuntura cinética como efeito potencializador dos elementos moduladores do movimento no tratamento de lesões desportivas. Fisioter Bras 2004 mar-abr; 5(2):111-8.

27. Wadsworth L T. Acupuncture in sports medicine. Curr Sports Med Rep 2006 Feb; 5(1):1-3.

28. Barela J A. Estratégias de Controle em Movimentos Complexos: Ciclo Percepção - Ação no Controle Postural. Rev Paul de Educ Fís 2000; 3: 79-88. 
29. Papler PG et al. Reabilitação do joelho. In: Greve J.M. A. e Amattuzzi, M.M. Medicina de reabilitação aplicada à ortopedia e traumatologia. São Paulo: Roca, 1999.

30. Gemeo LH. Ignatti C. Acupuntura como Ferramenta Auxiliar do Aumento da Performance Desportiva. In: Anais do Simpósio Internacional de Ciências Integradas da UNAERP; 2004 ; Guarujá, Brasil. São Paulo: Universidade de Ribeirão Preto Campus Guarujá; 2004. p. 1-9.

31. Rossetto SC. Acupuntura nos Esportes. 1 ed. São Paulo: Phorte, 2009.

32. Pelham TW, Holt LE, Stalker R. Acupuncture in human Performance. J Strength Cond Res 2001 May;15(2):266-71.

33. Myamoto T. Acupuncture treatment for muscle injury. Japa Journ of Phys Fit and Spor Med 1997; 43(2):39-41.

34. Fry AC, Kraemer KJ. Resistance exercise overtraining and overreaching: neuroendocrine responses. Sports Med 1997 Feb;23(2): 106-29.

35. Frasca L. Desempenho na Ponta da Agulha. Rev Farmac 2011 out-nov:44-5.

36. Costa V. Acupuntura Previne Lesões e ajuda a melhorar o Desempenho nas Pistas [periódico na internet]. 2013 [acesso em 22 jan 2017] Disponível em: http://blogs.oglobo.globo.com/pulso/post/acupuntura-previne-lesoes-ajudamelhorar-desempenho-nas-pistas-500798.html.

37. Luna M. Os Benefícios da Acupuntura no Esporte. 2016 [acesso em 22 jan 2017] Disponível em: http://www.ibramrp.com.br/noticia/49/os-beneficios-daacupuntura-no-esporte.

38. Akimoto T, Nakahori C, Aizawa K, Kimura F, Fukubayashi T, Kono I. Acupuncture and responses of imunoligic and endocrine markers during competition, Med Sci in Sports Exerc 2003 Ago;35(8):1296-1302.

39. Bopp-Limoge C. L’acupuncture Permet-elle D`ameliorer les Performances Sportives Stude Personnelle a Propos de 35 Sportifs de haut niveau. These Medicine 1998; 117:215.

40. Lin ZP et al. Effects of acupuncture stimulation on recovery ability for male elite basketball athletes. Amer Jour of Chin Med 2009; 37(3):471-81. 
41. Lin, ZP et al. Effect of auricular acupuncture on oxygen consumption of boxing athletes. Chin Med Jour 2009; 22(13):1587-90.

42. Dhillon $\mathrm{S}$ et al. The acute effect of acupuncture on 20-km cycling performance. Clin Jour of Spo Med 2008; 18(1):76-80.

43. Hubscher $\mathrm{M}$ et al. Immediate effects of acupuncture on strength performance: a ramdomized, controlled crossover trial. Euro Jour of Appli Physi 2010; 110(2):353-58.

44. Geng $\mathrm{L} J$ et al. Investigation on the effects of ear acupressure on exerciseinduced lactic acid levels and the implications for athletic training. Amer Jour of Acupu 1995; 23(4).

45. Fonseca LP, Lessa JFM. Efeito da Aplicação da Acupuntura na Resistência Muscular Localizada de Membros Superiores em Praticantes de Exercício Resistido. Brasília. Monografia [Graduação em Fisioterapia] - Centro Universitário de Brasília; 2011.

46. Belmiro H, Vicentini D, Camilotti CM. Efeitos da Acupuntura no Desempenho Motor de Atletas. Rev Fac Educ Fis 2013 jul-set; 11(3):176-91.

47. Angeli AL, Fernandes JGJ, Luna SPL. Acupuncture Applied Equine Sports Medicine, Ver Acad 2007 Jul-Set;5(3):325-33.

48. Staud R. Mechanisms of acupunture analgesia: effective therapy for musculoskeletal pain? Curr rheumatol Rep. 2007 Dec;9(6):473-81

49. Bucinskaite V, Lundeberg T, Stenfors C, Ekblom A, Dahlin L, Theodorsson E. Effects of electro-acupuncture and pysical exercice on regional concentrations of neuropeptides in rat brain, Brain research 1994 Dec;666(1): 128-32.

50. Shang C. Prospective tests on biological models of acupuncture. Evid Based Complement Alternat Med. 2009 Mar; 6(1):31-9.

51. Bowsher D. Mechanisms of acupuncture. In: Filshie J, White A. editors. Medial Acupuncture: a western scientific approach. London: Churchill Livingstone; 1988:69-82.

52. Ma SX. Neurobiology of acupuncture: Toward CAM. Evid Based Complement Alternat Med. 2004 Jun;1(1):41-7. 
53. Li J, Wang Q, Liang H, Dong H, Li Y, Ng EH, et al. Biophysical characteristics of meridians and acupoints: a systematic review. Evid Based Complement Alternat Med. 2012;2012793841.

54. Cardeal EL, Oliveira KB, Aoki MN, Amarante MK, Oliveira CEC, Suzuki S, et al. Aspectos imunológicos da acupuntura. Biosaúde 2005 Dez:7(1/2):49-60.

55. Szabó MVRS, Bechara GH. Acupuntura: Bases Científicas e Aplicações. Ciência rural 2001:31(6):1091-99

56. Saad M. A Medicina tradicional chinesa tem base científica?. Einstein: Educ Contin Saúde 2008: 6(3):122-6.

57. Alvarenga TF, Amaral CG, Steffen CP. Ação da acupuntura na neurofisiologia da dor: revisão bibliográfica. Rev Amazôn Scienc \& Helth 2014 Out/Dez:2(4):29-36.

58. Pereira FAO. Evidências científicas da ação da acupuntura. Perspectivas 2005 jan/jul:4(7):88-105.

59. Taffarel MO, Freitas PMC. Acupuntura e analgesia: aplicações clínicas e principais acupontos. Ciênc Rural 2009 Dez:39(9): 2665-72.

60. Lopes LF, Lopes MC, Fialho FAP, Gonçalves AL. Sistema de conhecimento para diagnóstico em acupuntura:uma modelagem usando o CommonKADS. Gest Prod 2010 Dez:x(x):1-15.

61.Zhu J, Arsovska B, Kozovska K. Acupuncture treatment for sports injury hamstring muscles group, Inter J Clin Exper Med Sci 2017 Nov;3(6):71-3.

62. Vickers AJ, Cronin AM, Maschino AC, Lewith G, MacPherson H, Foster NE, et al. Acupuncture for chronic pain individual patient data meta-analysis, Arch Intern Med 2012 Oct;172(19):1444-53.

63. Toda S. Effect of acupuncture on carnitine for skeletal muscle fatigue, Chin Med 2012 Jan;3:9-12.

64. Lopes SS, Mota MPG. Influência da acupuntura no limiar de percepção dolorosa de musculatura submetida a esforço repetitivo, $\mathrm{Br}$ J Pain 2018 julset;1(3):207-11.

65. Lee, SH, Chung, SH, Lee, JS, Kim, SS, Shin, HD, Lim, BV, et al. Effects Acupunturaq on the 5-hydroxytryptamine synthesis and tryptophan hydroxylase 
expression in dorsal in the dorsal raphe of exercice rats. Neurocienses Letters 2002 Oct;332(1): 17-20.

66. Zyloney CE, Jensen K, Polich G, Loiotile RE, Cheetham A, LaViolette PS, et al. Imaging the functional connectivity of the Periaqueductal Gray during genuine and sham electroacupuncture treatment. Mol pain. 2010 Nov;6:80

67. Minori AET, Mejia DPM. Atuação da Acupuntura para o Tratamento de LER/DORT no Ombro. Goiânia. Monografia [Pós Graduação em Acupuntura] Faculdade Ávila; 2007.

68. Brum KN, Alonso, AC, Brech GC. Tratamento de massagem e acupuntura em corredoresrecreacionais com síndrome do piriforme. Arq Cienc Sau 2009 abrjun; 16(2): 62-6.

69. Hongwen S. Clinical Observation on Acupuncture Treatment of Piriformis Syndrome. J Tradit Chin Med. 2003 mar; 23(1):38-9.

70. Rocha TBX, Vilela Junior GB, Martins GC, Manzatto L, Grande AJ. Análise Comparativa Eletromiográfica do Reto Femoral em Isometria na Posição Inferior do Agachamento Wall Slide, antes e após a Aplicação da Acupuntura no Ponto ST45. Rev Bras Cien e Mov 2012 jul; 20(4):92-8.

71. Pinheiro RG, Mejia DPM. Efeito da Acupuntura na Melhora do Paciente com Quadro Álgico de Lesão de Menisco Medial. Goiânia. Monografia [Pós Graduação em Acupuntura] - Faculdade Ávila; 2012.

72. Nunes EA, Mejia DPM. Tratamento de Acupuntura para Combater Dores nos Ombros. Goiânia. Monografia [Pós Graduação em Acupuntura] - Faculdade Ávila; 2012.

73. Parisotto D. Efeito Imediato da Aplicação da Acupuntura na Dor Muscular Tardia e na Capacidade de Contração Muscular. Curitiba. Dissertação [Mestrado em Fisiologia] - Universidade Federal do Paraná; 2014.

74. Tolentino F. Efeito de um Tratamento comAuriculoterapia na Dor, Funcionalidade e Mobilidade de Adultos com Dor Lombar Crônica. Rio Claro. Dissertação [Mestrado em Desenvolvimento Humano e Tecnologias] Universidade Estadual Paulista - UNESP; 2016.

Eingereicht: April 2019. 
Genehmigt: Oktober 2019. 